\title{
WCET-Aware Dynamic I-Cache Locking for a Single Task
}

\author{
WENGUANG ZHENG, Tianjin University of Technology \\ HUI WU, The University of New South Wales \\ QING YANG, The University of Rhode Island
}

\begin{abstract}
Caches are widely used in embedded systems to bridge the increasing speed gap between processors and off-chip memory. However, caches make it significantly harder to compute the worst-case execution time (WCET) of a task. To alleviate this problem, cache locking has been proposed. We investigate the WCETaware I-cache locking problem and propose a novel dynamic I-cache locking heuristic approach for reducing the WCET of a task. For a nonnested loop, our approach aims at selecting a minimum set of memory blocks of the loop as locked cache contents by using the min-cut algorithm. For a loop nest, our approach not only aims at selecting a minimum set of memory blocks of the loop nest as locked cache contents but also finds a good loading point for each selected memory block. We propose two algorithms for finding a good loading point for each selected memory block, a polynomial-time heuristic algorithm and an integer linear programming (ILP)-based algorithm, further reducing the WCET of each loop nest. We have implemented our approach and compared it to two state-of-the-art I-cache locking approaches by using a set of benchmarks from the MRTC benchmark suite. The experimental results show that the polynomial-time heuristic algorithm for finding a good loading point for each selected memory block performs almost equally as well as the ILP-based algorithm. Compared to the partial locking approach proposed in Ding et al. [2012], our approach using the heuristic algorithm achieves the average improvements of 33\%, 15\%, 9\%, 3\%, 8\%, and 11\% for the 256B, $512 \mathrm{~B}, 1 \mathrm{~KB}, 4 \mathrm{~KB}, 8 \mathrm{~KB}$, and $16 \mathrm{~KB}$ caches, respectively. Compared to the dynamic locking approach proposed in Puaut [2006], it achieves the average improvements of $9 \%, 19 \%, 18 \%, 5 \%, 11 \%$, and $16 \%$ for the $256 \mathrm{~B}$, $512 \mathrm{~B}, 1 \mathrm{~KB}, 4 \mathrm{~KB}, 8 \mathrm{~KB}$, and $16 \mathrm{~KB}$ caches, respectively.
\end{abstract}

Categories and Subject Descriptors: B.3.3 [Performance Analysis and Design Aids]: Worst-case Analysis General Terms: Algorithms, Performance

Additional Key Words and Phrases: Worst-case execution time, dynamic I-cache locking, minimum cut

ACM Reference Format:

Wenguang Zheng, Hui Wu, and Qing Yang. 2017. WCET-aware dynamic I-cache locking for a single task. ACM Trans. Archit. Code Optim. 14, 1, Article 4 (March 2017), 26 pages.

DOI: http://dx.doi.org/10.1145/3046683

\section{INTRODUCTION}

Caches (I-cache and D-cache) are effective to bridge the speed gap between processors and off-chip memory. With the utilization of caches, the execution time of a task can be significantly reduced. Since caches are managed by hardware, it is difficult to know at

Authors' addresses: W. Zheng, School of Computer and Communication Engineering, Tianjin University of Technology, 391 Bin Shui Road, Xi Qing District, Tianjing 300384, China; email: wenguangz@tjut.edu.cn; H. Wu, School of Computer Science and Engineering, The University of New South Wales, Sydney, NSW 2052, Australia; email: huiw@cse.unsw.edu.au; Q. Yang, Department of Electrical, Computer \& Biomedical Engineering, The University of Rhode Island, Kelley Hall, 4 East Alumni Ave., Kingston, RI 02881, USA; email: qyang@ele.uri.edu.

Permission to make digital or hard copies of part or all of this work for personal or classroom use is granted without fee provided that copies are not made or distributed for profit or commercial advantage and that copies show this notice on the first page or initial screen of a display along with the full citation. Copyrights for components of this work owned by others than ACM must be honored. Abstracting with credit is permitted. To copy otherwise, to republish, to post on servers, to redistribute to lists, or to use any component of this work in other works requires prior specific permission and/or a fee. Permissions may be requested from Publications Dept., ACM, Inc., 2 Penn Plaza, Suite 701, New York, NY 10121-0701 USA, fax +1 (212) 869-0481, or permissions@acm.org.

(c) 2017 ACM 1544-3566/2017/03-ART4 $\$ 15.00$

DOI: http://dx.doi.org/10.1145/3046683 
compile time whether a memory access is a cache hit or not. Therefore, caches make it significantly harder to compute the worst-case execution time (WCET) of a task.

Cache locking is an effective technique for alleviating the unpredictability problem of memory accesses. If an instruction or a data item is locked into the I-cache or the D-cache, it will not be replaced. As a result, each access to a locked instruction or data item is a cache hit, taking one cycle. Furthermore, cache locking can reduce the WCET of a task, as we can lock the memory blocks on the longest paths of the task to reduce the total access time of those memory blocks.

Cache locking approaches can be classified into two categories: static cache locking and dynamic cache locking. For static cache locking, a locked memory block of a task remains locked during the entire execution of the task. Therefore, the memory blocks mapped to the same location in a cache cannot be locked simultaneously. For dynamic cache locking, a locked memory block is unlocked after its live range expires. Thus, different memory blocks can be locked into the same location of a cache if their live ranges do not overlap, leading to more efficient utilization of a cache.

For static cache locking, the major task is to select a set of memory blocks to be locked into a cache. For dynamic cache locking, there is another major task: determining a loading point for each selected memory block. A selected memory block is loaded and locked into the cache at its loading point. To reduce the WCET of a task, we need to select the memory blocks on the worst-case execution path as locked cache contents. The longest path may change after some memory blocks on the longest path are locked into the cache. For this reason, all previous cache locking approaches iteratively select the memory blocks on the longest path to be locked into the cache. However, selecting memory blocks on a single longest path does not necessarily minimize the WCET of a task.

In this article, we investigate the WCET-aware I-cache locking problem for a single task. Given a task, our objective is to select a set of memory blocks of the code of the task and find a loading point for each selected memory block such that the WCET of the task is minimized after loading and locking the selected memory blocks into the I-cache at their loading points. We make the following major contributions:

(1) We propose a novel, min-cut-based, dynamic I-cache locking heuristic approach and two algorithms for finding a good loading point for each selected memory block: a polynomial-time heuristic algorithm and an integer linear programming (ILP)-based algorithm. Unlike all previous I-cache locking approaches that consider only the longest path, our approach considers a subgraph that contains not only the longest path but also all other paths whose lengths are close to the longest path length, and it selects a minimum set of memory blocks of the code of the loop as locked cache contents to reduce the maximum length of the whole subgraph.

(2) We have implemented our dynamic I-cache locking approach and compared it to the two state-of-the-art approaches, namely the partial I-cache locking approach proposed in Ding et al. [2012] and the longest path-based dynamic I-cache locking approach proposed in Puaut [2006], by using a set of benchmarks from the MRTC benchmark suite [Burger and Austin 1997]. The experimental results show that the polynomial-time heuristic algorithm for finding loading points performs almost equally as well as the ILP-based algorithm. Compared to the partial locking approach proposed in Ding et al. [2012], our approach using the polynomial-time heuristic for finding a good loading point for each selected memory block achieves the average improvements of of $33 \%, 15 \%, 9 \%, 3 \%, 8 \%$, and $11 \%$ for the $256 \mathrm{~B}, 512 \mathrm{~B}$, $1 \mathrm{~KB}, 4 \mathrm{~KB}, 8 \mathrm{~KB}$, and $16 \mathrm{~KB}$ caches, respectively. Compared to dynamic locking proposed in Puaut [2006], it achieves the average improvements of 9\%, 19\%, 18\%, 5\%, $11 \%$, and $16 \%$ for the $256 \mathrm{~B}, 512 \mathrm{~B}, 1 \mathrm{~KB}, 4 \mathrm{~KB}, 8 \mathrm{~KB}$, and $16 \mathrm{~KB}$ caches, respectively. 
This article is a major extension of previously published work [Zheng and Wu 2014]. Compared to Zheng and $\mathrm{Wu}$ [2014], this article proposes a new approach to loop nest locking, two new algorithms for finding a good loading point of each selected memory block, one polynomial-time heuristic algorithm, and one ILP-based algorithm, and provides new experimental results.

The rest of this article is organized as follows. Section 2 gives a survey of the related work. Section 6.3 presents a motivational example. Section 4 describes the system model and key definitions. Section 5 proposes our algorithm for selecting memory blocks of a nonnested loop as locked I-cache contents. Section 6 presents our approach to loop nests and two algorithms for finding a good loading point for each selected memory block. Section 7 shows the experimental results and analysis, and Section 8 concludes the article.

\section{RELATED WORK}

Many cache locking approaches have been proposed to reduce the WCET or the averagecase execution time (ACET) of a task. Anand and Barua [2009] propose a static I-cache locking approach to reduce the ACET of a task. The approach introduces a cost-benefit model to approximate the net benefit of locking a memory block into the I-cache and iteratively selects a memory block with the highest benefit as locked cache contents until the I-cache is full or no memory block has a net benefit. Liang and Mitra [2010] explore the static I-cache locking problem and propose an optimal algorithm and a heuristic approach that use the temporal reuse profile to determine the most beneficial memory blocks to be locked into the I-cache to reduce the ACET of a task. Liu et al. [2012] propose two static I-cache locking approaches and one dynamic I-cache locking approach that aim at minimizing the ACET of a task by exploring the probability profile information, proving that the ACET-aware I-cache locking problem is NP-hard. Qiu et al. [2014] present a branch prediction-directed dynamic I-cache locking approach to improve the ACET of a task through cache conflict miss reduction. The approach partitions the control flow graph $(\mathrm{CFG})$ of a program into disjoint execution regions and selects the most profitable memory blocks as locked cache contents by calculating the locking profit of each region. At runtime, locking routines are prefetched into a small high-speed buffer. The predetermined cache locking contents are loaded and locked at specific execution points during program execution. Anand and Barua [2015] investigate the problem of reducing the ACET of a task by using instruction cache locking and propose two heuristic algorithms: one using static cache locking and the other using dynamic cache locking.

Campoy et al. [2003] compare static cache locking and dynamic cache locking using a genetic algorithm, and they point out that static cache locking is more predictable and that dynamic cache locking shows better improvements in most cases. Puaut and Arnaud [2006] propose a dynamic I-cache locking approach that partitions a program into a set of regions. For each region, there is a loading point. Puaut [2006] presents two dynamic I-cache locking algorithms. One is a greedy algorithm, and the other is a genetic algorithm. A cost function is used for each loop to determine whether its preheader should be selected as a loading point. However, the cost function is not well defined.

Falk et al. [2007] propose a static I-cache locking approach that aims at minimizing the WCET of a task by iteratively reducing the longest path length of the task. It uses an execution flow graph (EFG) to model the possible execution paths and selects the basic block on the current longest path with the largest benefit to be locked into the cache. Liu et al. [2009] investigate the I-cache locking problem and formulate the problem using an execution flow tree (EFT) and a linear programming model. For a subset of the problems with certain properties, they propose polynomial-time optimal algorithms. Furthermore, they prove that the general problem is NP-hard. Plazar 
et al. [2012] propose an ILP-based, static I-cache locking algorithm for minimizing the WCET of a task. Ding et al. [2012] point out that full cache locking may cause more cache misses that would have a negative effect on WCET reduction and propose a partial I-cache locking mechanism to lock parts of the I-cache.

Ding et al. [2014] propose a WCET-aware, dynamic I-cache locking approach for a single task. The approach uses ILP to determine the locking slots for each loop and selects the most profitable memory blocks to fill these slots. The locking benefit of a memory block $m$ is defined as benefit ${ }_{m}=\left(L A T_{\text {miss }}-L A T_{h i t}\right) \times f r e q_{m}$, where $L A T_{\text {miss }}$ and $L A T_{h i t}$ are the cache miss latency and the cache hit latency, respectively, and freqm is the execution frequency of the memory block $m$ on the worst-case path. However, the approach is incomplete and seriously flawed. First, it assumes that the execution frequency of a memory block $m$ on the worst-case path is known. However, this assumption is not valid because the longest path may change after a set of memory blocks are locked. Second, it does not address the problem of determining a good locking point for each selected memory block.

Several approaches to the D-cache locking problem have been proposed. Vera et al. [2003] propose an approach that combines compile-time cache analysis with data cache locking to estimate the worst-case memory performance (WCMP) in a safe, tight, and fast way. To obtain predictable cache behavior, the approach first locks the cache for those parts of the code where the static analysis fails. To minimize the performance degradation, the approach loads the cache with the data likely to be accessed. Zheng and $\mathrm{Wu}$ [2015] propose two D-cache locking approaches that aim at minimizing the WCET of a task. The first approach formulates the problem as a global ILP problem that simultaneously selects a near-optimal set of variables as the locked cache contents and allocates them to the D-cache. The second one iteratively constructs a subgraph of the CFG of the task where the lengths of all paths are close to the longest path length and uses an ILP formulation to select a near-optimal set of variables in the subgraph as the locked cache contents and allocate them to the D-cache. Zheng and Wu [2017] also propose two improved approaches that consider the individual sections of nonscalar variables in different cache sets as locked cache contents and a new D-cache allocation algorithm.

Several approaches have been proposed to integrate task scheduling and cache locking. Campoy et al. [2001] propose a genetic algorithm for the problem of selecting instructions to be locked into the I-cache to reduce the response time of multitasks. Puaut and Decotigny [2002] investigate static I-cache locking for multitask real-time systems. They propose two algorithms: one aiming at minimizing the CPU utilization and the other attempting to minimize the interferences between tasks. Campoy et al. [2002] propose a dynamic I-cache locking approach for multitask systems. The approach uses the response time analysis approach proposed in Busquets-Mataix et al. [1996] for the schedulability test and combines the schedulability analysis with cache locking using a genetic algorithm to improve the performance of the I-cache on a multitasking, preemptive real-time system. Liu et al. [2010] propose an approach that combines cache locking with task assignment to reduce the WCETs of a set of tasks on a multiprocessor system with two levels of caches. The approach applies cache locking to both the I-cache and D-cache by using the algorithms proposed in Liu et al. [2009] and Vera et al. [2003] to reduce the WCET of each task, and it optimizes the task assignment considering the cache size.

\section{A MOTIVATIONAL EXAMPLE}

In this section, we use an example to illustrate the key ideas of our approaches and compare it to three state-of-the-art approaches to the WCET-aware I-cache locking problem for a single task. The three approaches are the static full cache locking approach proposed in Falk et al. [2007], the longest path-based dynamic cache locking approach proposed in Puaut [2006], and the partial cache locking approach proposed in 


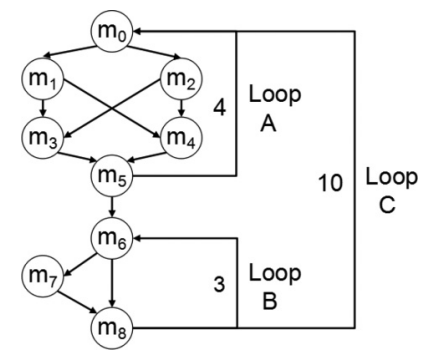

Fig. 1. CFG of the motivation example.

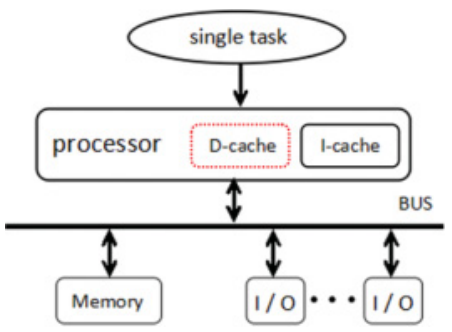

Fig. 2. System model.

Ding et al. [2012]. Consider a task with the CFG shown in Figure 1. The task consists of three loops where the loop A and the loop B are nested in the loop C. The numbers of iterations of the loop $\mathrm{A}$, the loop $\mathrm{B}$, and the loop $\mathrm{C}$ are 4, 3, and 10, respectively. For ease of descriptions, we make the following assumptions:

(1) The preheader of each loop is empty.

(2) All of the basic blocks are mapped to the same set of the I-cache.

(3) Each basic block is exactly one memory block.

(4) The associativity of the I-cache is 4.

(5) If a basic block is in the I-cache, its execution time is 1 cycle. Otherwise, its execution time is 30 cycles.

(6) The time for fetching and locking one memory block is 30 cycles.

Figure 3 illustrates the locked basic blocks and their loading points computed by the three approaches and our approach, where each small circle denotes a preheader containing instructions for loading and locking the relevant memory blocks. For example, Figure 3(a) shows that the basic blocks $m_{0}, m_{1}, m_{3}$, and $m_{5}$ are selected as the locked contents by the static full cache locking approach, and these four basic blocks are loaded and locked at the preheader of the loop C.

\subsection{Static Full Cache Locking}

Consider the approach proposed in Falk et al. [2007], which iteratively finds the longest path and selects the basic block with the largest benefit on the longest path as locked cache contents. Obviously, the basic blocks of the loop A have larger benefits due to its larger number of iterations. The four basic blocks $m_{0}, m_{1}, m_{3}$, and $m_{5}$ may be subsequently selected as locked cache contents at the preheader of the loop C as shown in Figure 3(a). Since this approach uses static cache locking, the selected basic blocks are fetched and locked only once. Therefore, the time taken to fetch and lock the selected basic blocks is $30 * 4=120$. As a result, the WCET of the task is 5,300 .

\subsection{Longest Path-Based Dynamic Cache Locking}

Using the longest path-based dynamic cache locking algorithm proposed in Puaut [2006], the selected basic blocks are loaded and locked at the preheaders of the loop $\mathrm{A}$ and the loop B as shown in Figure 3(b). For the loop A, $m_{0}, m_{1}, m_{4}$, and $m_{5}$ may be subsequently selected as locked cache contents, as they have the largest frequencies on the current longest paths. When the loop A terminates, all cache lines occupied by its memory blocks are released. Therefore, the entire loop B will be locked. The memory blocks $m_{0}, m_{1}, m_{4}$, and $m_{5}$ will be fetched and locked 10 times at the preheader of the loop A, and the three memory blocks of the loop B will also be fetched and locked 


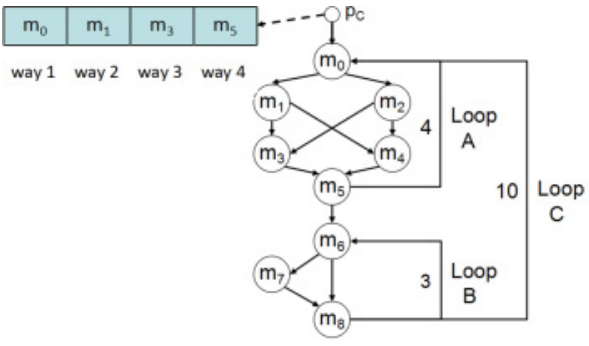

(a) Static full cache locking

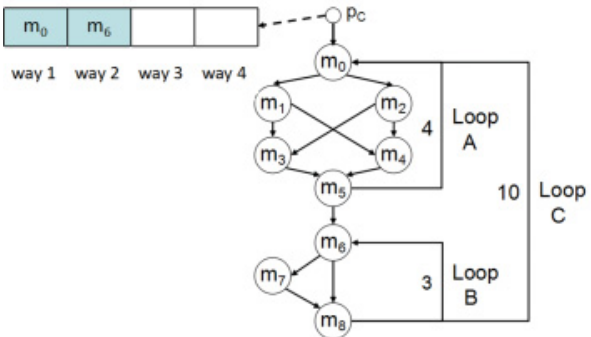

(c) Partial cache locking

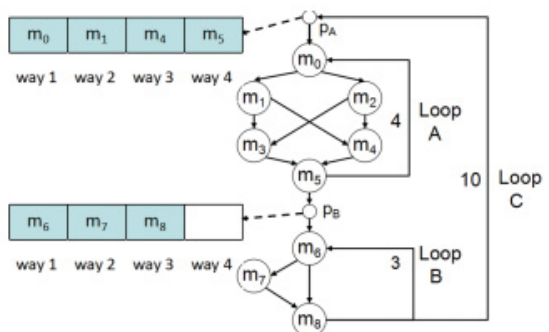

(b) Longest path-based dynamic cache locking

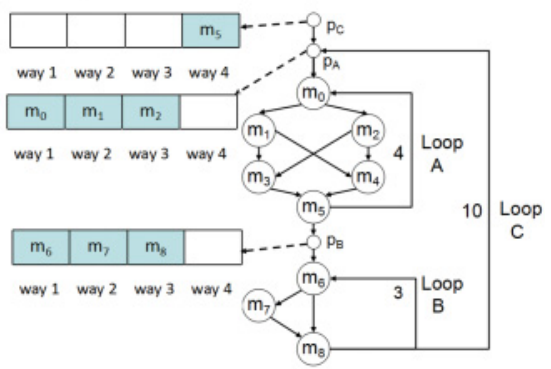

(d) Our approach

Fig. 3. Locked basic blocks and loading points by different approaches.

10 times at the preheader of the loop B. Thus, the time taken to fetch and lock the selected memory blocks is 2,100 , and the WCET of the task is 4,670 .

\subsection{Partial Cache Locking}

Using the partial cache locking algorithm proposed in Ding et al. [2012], $m_{0}$ and $m_{6}$ may be selected as locked cache contents at the preheader of the loop $\mathrm{C}$ as shown in Figure 3(c). The other two cache lines are kept free so that the memory accesses to $m_{7}$ and $m_{8}$ will be hit after the first cache misses during the execution of the loop B. For the loop A, each iteration will cause three cache misses. Since only two memory blocks are locked, the time for fetching and locking these two memory blocks is 60 , and the WCET of the task is 4,370 .

\subsection{Our Approach}

To find a better solution, our dynamic cache locking approach aims at finding a minimum set of memory blocks as locked cache contents and finds a good loading point for each selected memory block. First, our approach finds the minimum node cuts of the CFG of each loop of a loop nest without back edges from the innermost loops to the outermost loop and selects the memory blocks in the minimum node cuts as locked cache contents considering the cache capacity constraints. For the loop A, the minimum node cuts $\left\{m_{0}\right\},\left\{m_{1}, m_{2}\right\}$, and $\left\{m_{5}\right\}$ are selected. For the loop B, all three basic blocks are selected. Second, our approach moves the loading points of the selected memory blocks to the preheaders of the outer loops to further reduce the WCET of the loop nest. Initially, the loading point of a selected memory block is at the preheader of the loop that contains it directly. Later, a loading point may be moved to the preheader of an outer loop if the motion of the loading point results in a smaller WCET of the whole loop nest. In this example, our approach moves the loading point of $m_{5}$ from the preheader of the loop A to the preheader of the loop $\mathrm{C}$ to further reduce the WCET of 
the entire loop nest as shown in Figure 3(d). Notice that after the loading point of a selected memory block is moved to the preheader of an outer loop, the live range of the memory block is extended to the whole outer loop. By our approach, the WCET of the task is 3,240 .

\section{SYSTEM MODEL AND DEFINITIONS}

We investigate the WCET-aware dynamic I-cache locking problem for a single task. The target processor has an I-cache and a D-cache as shown in Figure 2. The I-cache is an $n$-way set associative cache with $k$ sets. Each set has a set number between 0 and $k-1$, and all sets have the same size. We do not consider the D-cache locking problem.

A basic block is a sequence of instructions with only one entry and one exit. Each basic block is mapped into one or more sets of the I-cache. The main memory is partitioned into memory blocks such that each memory block is mapped to exactly one cache line. The basic locking unit is a cache line. If an instruction is locked in the I-cache, it takes one cycle to fetch the instruction. Otherwise, it takes $c$ cycles.

Since it is not beneficial to lock a memory block that is not in any loop, we consider individual loop nests only. Notice that after a loop nest finishes, all of its locked memory blocks can be unlocked. Therefore, each loop nest can use the entire I-cache.

For each loop nest, we define a loop nest tree as follows.

Definition 4.1 (Loop Nest Tree). Given a loop nest, its loop nest tree is weighted tree $T=<L, W, E>$, where, $L=\left\{L_{i}: L_{i}\right.$ is a loop in the loop nest $\}, W=\left\{w_{i}: w_{i}\right.$ is the WCET of the loop $\left.L_{i}\right\}$, and $E=\left\{\left(L_{i}, L_{j}\right): L_{j}\right.$ is immediately nested in $\left.L_{i}\right\}$.

We use CFG to represent a task where each node has a weight, denoting the execution time of the corresponding basic block.

Given a loop nest $L$, we construct a weighted DAG $G_{i}$ for each loop $L_{i}$ of $L$ recursively as follows:

(1) Construct the loop nest tree of $L$.

(2) Construct the control flow graph $C F G(L)$ of $L$, where the weight of each node is the execution time of the corresponding basic block.

(3) For each loop $L_{i}$ of $L$ in reverse topological order of the loop nest tree, do the following:

(a) Let $G_{i}$ be the whole subgraph of $C F G(L)$ that represents $L_{i}$.

(b) Remove all back edges of $G_{i}$.

(c) Shrink the whole subgraph of $C F G(L)$ that represents $L_{i}$ into two nodes: a preheader node and a loop node.

(d) Set the weight of the loop node to the WCET of $L_{i}$ as follows:

-If $L_{i}$ is a loop where the loop exit condition is checked at the beginning of each iteration, the WCET of $L_{i}$ is equal to $l_{\max }\left(G_{i}\right) * n\left(L_{i}\right)+w_{h}\left(L_{i}\right)$, where $l_{\text {max }}\left(G_{i}\right), n\left(L_{i}\right)$, and $w_{h}\left(L_{i}\right)$ are the length of the longest path of $G_{i}$ without the preheader node, the maximum number of iterations of $L_{i}$, and the execution time of the header of $L_{i}$, respectively. Otherwise, it is $l_{\max }\left(G_{i}\right) * n\left(L_{i}\right)$.

The weighted DAG of the loop nest $L$ is the weighted DAG of the root of the loop nest tree of $L$.

Definition 4.2 ( $x$-Spanning Graph). Given a weighted DAG $G$ and a positive constant $x$, the $x$-spanning graph $G(x)$ is a subgraph of $G$ where the length of each path is larger than $x$.

Given a weighted DAG $G$, its $x$-spanning graph can be computed in $O(e)$ time as shown in Algorithm 1, where $e$ is the number of edges in $G$. 


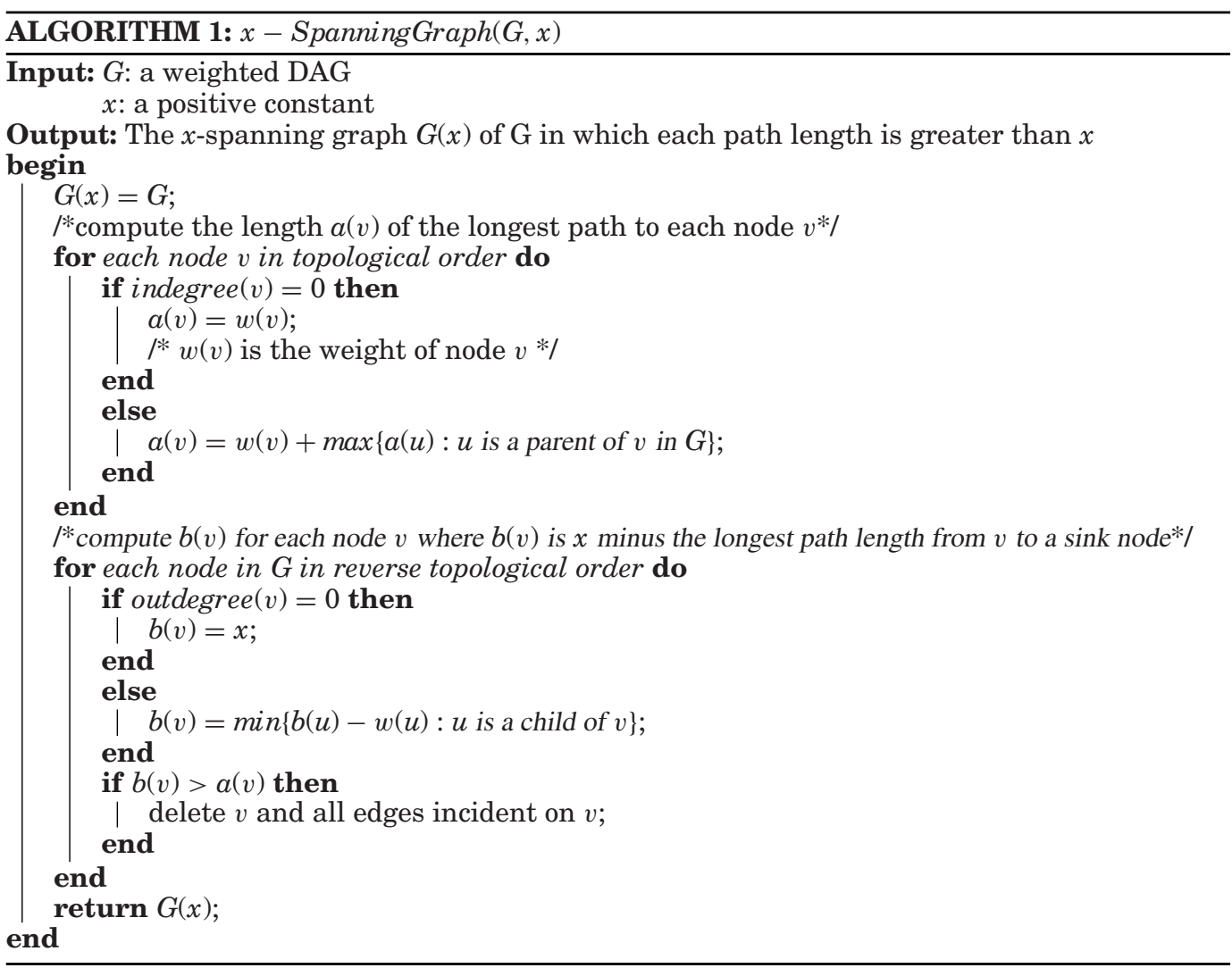

Definition 4.3 ( $y$-Projection Graph). Given an $x$-spanning graph $G(x)$ and a set $y$ of the I-cache, the $y$-projection graph $G(x, y)$ is a subgraph of $G(x)$ where the corresponding basic block or loop of each node has a memory block mapped into the set $y$ and the memory block has not been selected as locked cache contents.

The algorithm for constructing the $y$-projection graph $G(x, y)$ is shown in Algorithm 2. Its time complexity is $O(e)$, where $e$ is the number of edges in $G(x)$.

The WCET-aware, dynamic cache locking problem is formulated as follows. Given a task, select a set of memory blocks of code as locked cache contents, and for each selected memory block, find its loading point and address (cache line number) in the cache set to which it is mapped such that the WCET of the task is minimized. We propose a heuristic approach to this problem. After selecting a set of memory blocks to be locked into the I-cache, our approach stores the memory addresses of all selected memory blocks at the end of code section so that they can be locked into the I-cache efficiently. To lock the selected memory blocks into the corresponding cache lines and reduce the size of extra code for cache locking, we introduce a special instruction lock with the following format:

$$
\text { lock offset, start-addr, entries, }
$$

where start-addr is the start address of a sequence of memory blocks that are contiguously locked into the same set of the I-cache, entries is the number of memory blocks of the sequence, and offset is the offset in the I-cache of the first memory block of the sequence. For each memory block $M_{i}(i=0,1, \ldots$, entries -1$)$ specified by the three 


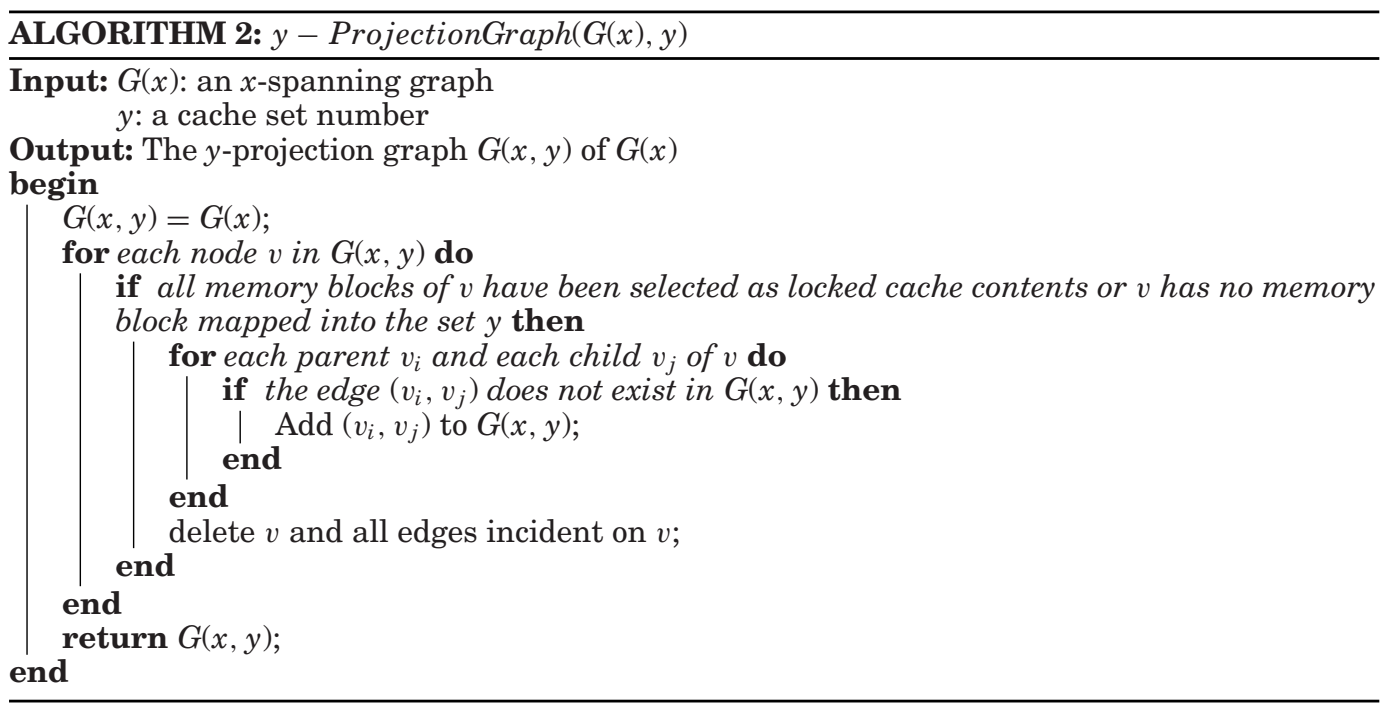

operands, the lock instruction locks $M_{i}$ into the (offset $\left.+i\right)$-th cache line of the corresponding cache set, updates the corresponding entry in the tag store, and sets the lock bit of the cache line. The lock instruction takes $c$ cycles for loading and locking each memory block, where $c$ is the cache miss penalty.

When a cache line is already locked, lock can still load a memory block into it. To prevent disrupting the mapping between basic blocks and memory blocks, we insert a procedure call instruction at the preheader of each loop. The instruction calls the corresponding loading procedure that is placed at the end of the task. The loading procedure contains a sequence of lock instructions for loading the selected memory blocks and locking them. The program point of a call to a loading procedure is called a loading point. Initially, each loading procedure is empty. After a set of memory blocks of a task is selected as locked cache contents, a sequence of zero or more lock instructions will be added to each loading procedure. If no lock instruction is added to a loading procedure, the corresponding call will be replaced by a nop instruction.

Give a set $S,|S|$ denotes the number of elements of $S$.

\section{I-CACHE LOCKING FOR A NON-NESTED LOOP}

Before presenting our approach to dynamic I-cache locking for a loop nest, we propose a min-cut-based algorithm for selecting the memory blocks of a nonnested loop as locked cache contents. Given a nonnested loop $L_{i}$, our algorithm aims at selecting a minimum set of memory blocks of the loop as locked cache contents such that the WCET of the loop is minimized. Our algorithm works as follows:

(1) Construct the weighted DAG $G_{i}$ of the loop as described in Section 4.

(2) Repeat the following steps for each set $y$ of the I-cache until the set $y$ is full or no more memory block can be selected as locked cache contents:

(a) Find the longest path length $l_{\max }\left(G_{i}\right)$ of $G_{i}$.

(b) Construct the $x$-spanning graph of $G_{i}$, where $x$ is $l_{\text {max }}\left(G_{i}\right)-c$, where $c$ is the off-chip memory access latency.

(c) Construct the $y$-projection graph of the $x$-spanning graph.

(d) Find a minimum node cut $N$ of the $y$-projection graph. 


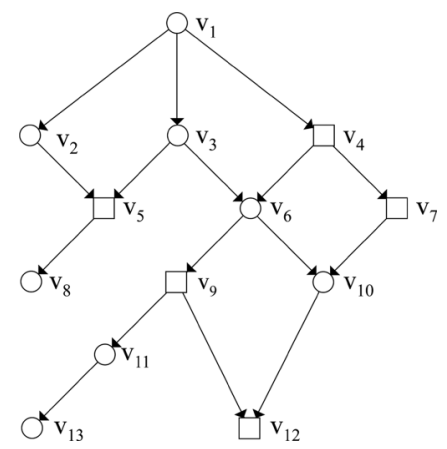

Fig. 4. A DAG $G$.

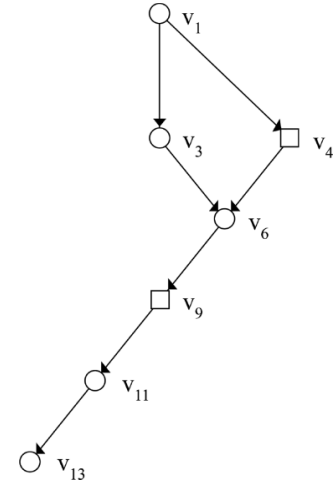

Fig. 5. G(150).

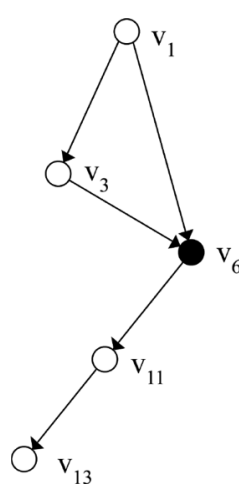

Fig. 6. G(150,0).

(e) If the size of $N$ is not less than the number of iterations of the loop, discard $N$ and the algorithm terminates. Otherwise, if the number of free cache lines of the set $y$ is not less than the size of $N$, do the following:

(i) For each node $v_{i}$ in $N$, select a memory block $m_{i}$ of its corresponding basic block with the largest execution time among all memory blocks of the basic block that has not been selected as locked cache contents.

(ii) Recalculate the execution times of $v_{i}$ and all basic blocks affected.

(iii) Update the weight of each node of $N$.

When the size of a node cut is not less than the number of iterations of the loop, locking the memory blocks of the corresponding basic blocks of the node cut does not reduce the WCET of the loop. Furthermore, the size of a node cut does not exceed the size of the previous node cut during the execution of our algorithm. Therefore, our algorithm terminates when the size of a node cut is not less than the number of iterations of the loop.

The details of our algorithm for a single loop are given in Algorithm 3. This algorithm returns $C_{y}\left(L_{i}\right)$ and size [], where $C_{y}\left(L_{i}\right)$ stores a set of the minimum cuts of the memory blocks of the loop $L_{i}$ and size[i] stores the number of the memory blocks of the loop $L_{i}$ that have been selected as locked cache contents in each cache set $i$. If $L_{i}$ does not contain a nested loop, all memory blocks in $C_{y}\left(L_{i}\right)$ will be locked. Otherwise, our approach to a loop nest will determine which minimum cuts in $C_{y}\left(L_{i}\right)$ are locked by comparing the benefit of locking a minimum cut and the benefit of moving a loading point from the preheader of an inner loop to the preheader of $L_{i}$.

We use an example to show how our algorithm works. Consider a weighted DAG $G$ shown in Figure 4. Assume that the I-cache has two sets, and each node (basic block) is exactly one memory block. The execution time of each basic block is 1 if it is locked into the I-cache. Otherwise, it is 30 . All circle nodes are mapped to set 0 , and all square nodes are mapped to set 1 . Next, we show how our algorithm selects the locked cache contents for set 0 . Based on the assumptions, the longest path length of the weighted DAG $\mathrm{G}$ is 180 and $x$ is 150 . In the first iteration, $G(150)$, the 150 -spanning graph, is constructed as shown in Figure 5. Based on $G(150)$, the 0-projection graph $G(150,0)$ of $G(150)$ is constructed as shown in Figure 6 . In $G(150,0)$, all nodes belong to set $0 ;\left\{v_{6}\right\}$ is a minimum node cut of $G(150,0)$. Therefore, $v_{6}$ is selected as the locked contents, and its weight is changed from 30 to 1 . Now, the longest path length of $G$ becomes 151 . In the second iteration, $G(121)$ is constructed as shown in Figure 7, and the 0-projection graph of $G(121)$ is constructed as shown in Figure 8. A minimum node cut of $G(121,0)$ is $\left\{v_{1}\right\}$. Hence, our algorithm selects $v_{1}$ as locked cache contents as shown in Figure 9. 


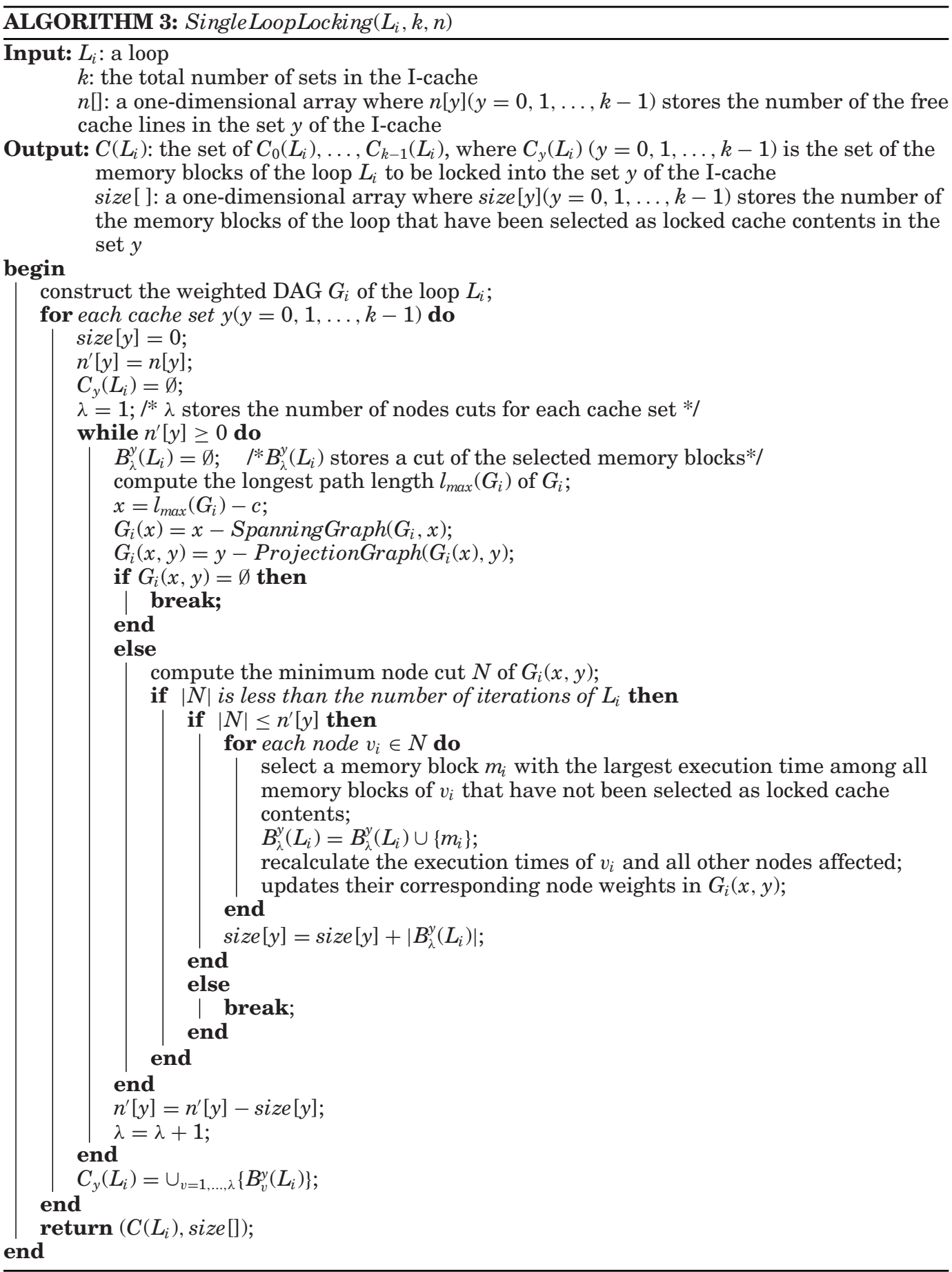




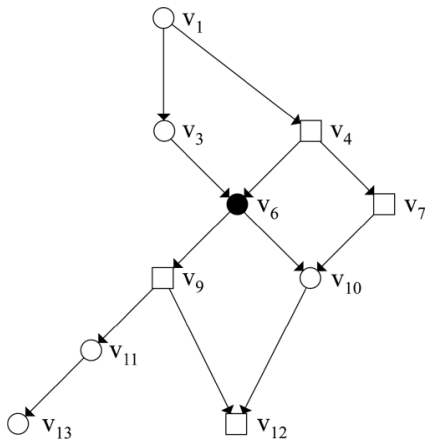

Fig. 7. G(121) with $v 6$ locked.

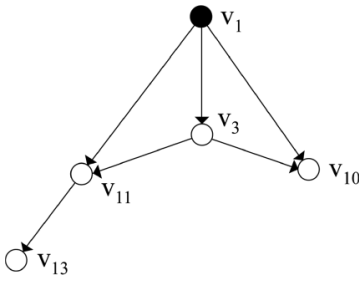

Fig. 8. G(121,0) with $v 6$ removed.

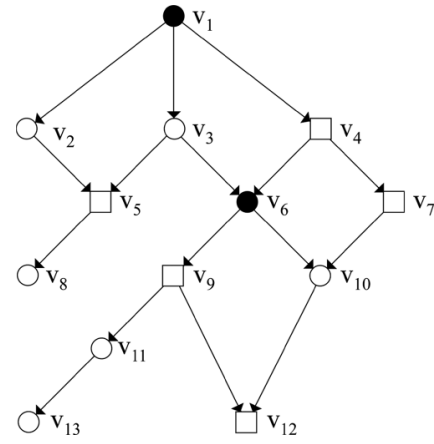

Fig. 9. The DAG G after locking $v 6$ and $v 1$.

Now, the longest path length of DAG $G$ is reduced to 122 . The same selection process is applied to square nodes to find locked cache contents for set 1.

Next, we analyze the time complexity of Algorithm 3. Clearly, the time complexity of one iteration of the outermost for loop is dominated by finding a minimum node cut $C$ of the $y$-projection graph. The maximum number of iteration of the while loop is $O(m)$, where $m$ is the number of memory blocks of the loop. Therefore, the time complexity of Algorithm 3 is $O(m t)$, where $t$ is the time complexity of finding a minimum node cut of a $y$-projection graph. The minimum node cut problem can be converted into the minimum edge cut problem in $O(e)$ time [Skiena 1998], where $e$ is the number of edges in the graph. Given a weighted DAG, the minimum edge cut can be found in $O\left(n e+n^{2} \log n\right)$ time [Stoer and Wagner 1997], where $n$ and $e$ are the number of nodes and the number of edges, respectively, of the DAG $G_{i}$. As a result, the time complexity of Algorithm 3 is $O\left(m n e+m n^{2} \log n\right)$.

\section{CACHE LOCKING FOR A LOOP NEST}

In this section, we propose an approach to the problem of the WCET-aware dynamic I-cache locking for a loop nest. The objective of cache locking for a loop nest is to select a set of memory blocks of the loop nest and determine both the loading point and the start address in the corresponding cache set for each selected memory block such that the WCET of the loop nest is minimized and the cache capacity constraints are satisfied. To facilitate descriptions, we introduce the notations shown in Table I.

Consider a loop nest $L$. For each cache set $y(y=0,1, \ldots, k-1)$, our approach starts with leaf loops and work toward the root of the loop nest tree of $L$. Each time, it selects an unprocessed loop $L_{i}$ with the maximum total number of iterations on the longest path of the weighted DAG of the loop nest $L$. If $L_{i}$ is an innermost loop, our approach uses Algorithm 3 to select a set $C_{y}\left(L_{i}\right)$ of the memory blocks of $L_{i}$ as locked cache contents and set their loading points to the preheader of $L_{i}$. Otherwise, our approach first evokes Algorithm 3 to select a set $C_{y}\left(L_{i}\right)$ of the memory blocks of $L_{i}$ as the candidates of locked cache contents and then calls our heuristic algorithm or our ILP-based algorithm to select a subset of the memory blocks in $C_{y}\left(L_{i}\right)$ as locked cache contents and a set $M_{y}\left(L_{i}\right)$ of previously memory blocks whose loading points are in the preheaders of the children of $L_{i}$, sets the loading points of all memory blocks in the subset of $C_{y}\left(L_{i}\right)$ to the preheader of $L_{i}$, and moves the loading points of all memory blocks in $M_{y}\left(L_{i}\right)$ from the preheaders of the children of $L_{i}$ to the preheader of $L_{i}$.

The objective of both the heuristic algorithm and the ILP-based algorithm is to select a subset of the memory blocks in $C_{y}\left(L_{i}\right)$ and a set $M_{y}\left(L_{i}\right)$ of the previously selected memory blocks whose loading points are at the preheaders of the children of $L_{i}$. The 
Table I. Notations

\begin{tabular}{|c|c|}
\hline Notation & Definition \\
\hline$s$ & Number of cache lines in each set of the I-cache \\
\hline$c$ & Time for loading and locking one memory block into the I-cache \\
\hline$I_{i}$ & Number of iterations of the loading points at the preheader of $L_{i}$ \\
\hline$c_{y}\left(L_{i}\right)$ & $\begin{array}{l}\text { Total number of cache lines needed to store all selected memory } \\
\text { blocks of the loop nest tree rooted at } L_{i} \text { in the cache set } y\end{array}$ \\
\hline$A_{y}\left(L_{i}\right)$ & $\begin{array}{l}\text { Set of memory blocks that are loaded and locked at the } \\
\text { preheader of a loop } L_{i} \text { in the set } y \text { of the I-cache }\end{array}$ \\
\hline$C H\left(L_{i}\right)$ & Set of children of a loop $L_{i}$ in the loop nest tree \\
\hline $\begin{array}{c}B_{v}^{y}\left(L_{i}\right)(y=0,1, \ldots, k-1 \text { and } \\
v=1, \ldots, \lambda)\end{array}$ & $\begin{array}{l}\text { Minimum cut of the memory blocks of } L_{i} \text { selected as the } \\
\text { candidates of locked cache contents by using Algorithm } 3 \text { for } \\
\text { each cache set } y\end{array}$ \\
\hline
\end{tabular}

memory blocks of the subset and $M_{y}\left(L_{i}\right)$ will be loaded and locked into the I-cache at the preheader of the loop $L_{i}$.

Our approach loads and locks the selected memory blocks into the I-cache at the preheaders of loops. The live range of a selected memory block spans the whole loop that loads and locks the memory block into the I-cache at its preheader. If a loading point of a memory block is moved from the preheader of a child of $L_{i}$ in the loop nest tree to the preheader of $L_{i}$, the live range of the memory block will be extended to the whole loop of $L_{i}$. For each pair of the memory blocks whose loading points are in the preheaders of the two children of $L_{i}$ in the loop nest tree, their live ranges are disjoint. Furthermore, for each memory block loaded at the preheader of $L_{i}$, its live range overlaps with that of each memory block loaded at the preheader of any child of $L_{i}$ in the loop nest tree.

To determine the number of the free cache lines in the cache set $y(y=0,1, \ldots, k-1)$, for each loop $L_{i}$, we introduce a set of variables as shown in Table I.

After our approach calls our heuristic algorithm or our ILP-based algorithm, $c_{y}\left(L_{i}\right)$ needs to be updated as follows:

$$
c_{y}\left(L_{i}\right)=\left|A_{y}\left(L_{j}\right)\right|+\max \left\{c_{y}\left(L_{j}\right): L_{j} \in C H\left(L_{i}\right)\right\} .
$$

After our approach selects a set of memory blocks as locked cache contents and finds a loading point for each selected memory block, it inserts lock instructions to the loading procedure of each loop $L_{i}$ to load and lock the selected memory blocks of $L_{i}$ into the I-cache. In each cache set $y(y=0,1, \ldots, k-1)$, all memory blocks locked at the preheader of each loop $L_{i}$ are stored contiguously in any order. Let $a d d r_{y}\left(L_{i}\right)$ be the start address (cache line number) of the first memory block locked into the set $y$ at the preheader of a loop $L_{i}$. For each loop $L_{i}, a d d r_{y}\left(L_{i}\right)$ is recursively calculated as follows:

$$
a d d r_{y}\left(L_{i}\right)=\left\{\begin{array}{lc}
0 & L_{i} \text { is an innermost loop } \\
\max \left\{a d d r_{y}\left(L_{j}\right)+\left|A_{y}\left(L_{j}\right)\right|: L_{j} \in C H\left(L_{i}\right)\right\} & \text { otherwise. }
\end{array}\right.
$$

The details of our approach to the WCET-aware, dynamic cache locking problem for a loop nest are shown in Algorithm 4. Our heuristic algorithm and ILP-based algorithm will be described in Sections 6.1 and 6.3, respectively.

\subsection{Heuristic Algorithm}

The objective of our heuristic algorithm is to select a subset of the memory blocks in $C_{y}\left(L_{i}\right)$ as locked cache contents and a set $M_{y}\left(L_{i}\right)$ of previously selected memory blocks whose loading points are in the preheaders of the children of $L_{i}$, set the loading points of all memory blocks in the subset of $C_{y}\left(L_{i}\right)$ to the preheader of $L_{i}$, and move the loading 


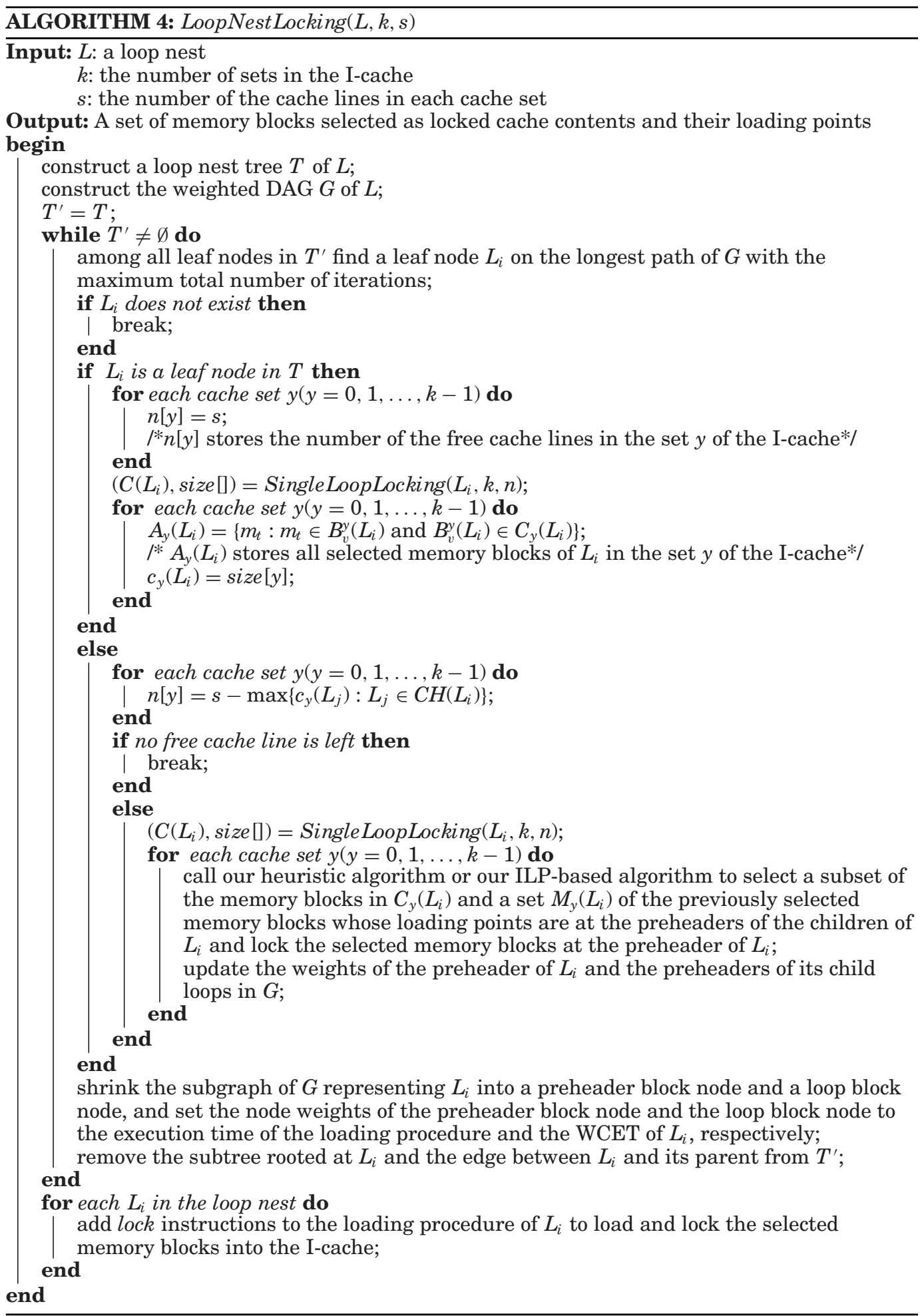


points of all memory blocks in $M_{y}\left(L_{i}\right)$ from the preheaders of the children of $L_{i}$ to the preheader of $L_{i}$, such that the WCET of $L_{i}$ is minimized.

For each cut $B_{v}^{y}\left(L_{i}\right) \in C_{y}\left(L_{i}\right)(v=1, \ldots, \lambda)$, let $R\left(B_{v}^{y}\left(L_{i}\right)\right)$ be the WCET reduction of $L_{i}$ by locking all memory blocks in $B_{v}^{y}\left(L_{i}\right)$ at the preheader of $L_{i}$. We define a benefit, denoted benefit $\left(B_{v}^{y}\left(L_{i}\right)\right)$, as follows:

$$
\operatorname{benefit}\left(B_{v}^{y}\left(L_{i}\right)\right)=\frac{R\left(B_{v}^{y}\left(L_{i}\right)\right)}{\left|B_{v}^{y}\left(L_{i}\right)\right|} .
$$

Our heuristic algorithm works iteratively. Each time, it finds a cut $B_{v}^{y}\left(L_{i}\right)$ from $C_{y}\left(L_{i}\right)$ with the maximum benefit and a set $S$ of the memory blocks in $\cup_{L_{j} \in C H\left(L_{i}\right)} A_{y}\left(L_{j}\right)$ with the maximum impact on the WCET reduction of the loop nest such that the size of the cache set $y$ used by the currently selected locked cache contents after moving the loading points of all memory blocks of $S$ to the preheader of $L_{i}$ does not exceed the size of the cache set $y$ used by the currently selected locked cache contents after locking the memory blocks of $B_{v}^{y}\left(L_{i}\right)$ at the preheader of $L_{i}$. If the memory blocks in $B_{v}^{y}\left(L_{i}\right)$ reduce the WCET of the loop nest more than the memory blocks in $S$ do, our heuristic algorithm selects all memory blocks in $B_{v}^{y}\left(L_{i}\right)$ as locked cache contents and locks them into the I-cache at the preheader of $L_{i}$. Otherwise, it moves the loading points of all memory blocks in $S$ to the preheader of $L_{i}$. When no loading point can be moved, our heuristic keeps selecting a cut with the maximum impact on the WCET reduction of the loop nest as locked cache contents. When no memory cut in $C_{y}\left(L_{i}\right)$ is left, our heuristic keeps moving a loading point with the maximum WCET reduction from the preheader of a child of $L_{i}$ in the loop nest tree to the preheader of $L_{i}$. The details of our heuristic algorithm are shown in Algorithm 5. This algorithm calls NoComparison() to handle the two special cases where either no loading point from the children of $L_{i}$ can be moved or no cut in $C_{y}\left(L_{i}\right)$ is left.

\subsection{Time Complexity Analysis}

First, we analyze the time complexity of our heuristic algorithm, Algorithm 5. Given a loop $L_{i}$, this algorithm either selects a cut from $C_{y}\left(L_{i}\right)$ or selects a set of memory blocks from $\cup_{L_{j} \in C H\left(L_{i}\right)} A_{y}\left(L_{j}\right)$ at each iteration. Therefore, the total number of iterations of the while loop is $O\left(n_{i}\right)$, where $n_{i}$ is the number of memory blocks of the loop $L_{i}$. The time complexity of computing the WCET of $L_{i}$ is $O\left(e_{i}\right)$, where $e_{i}$ is the number of edges in the weighted graph $G_{i}$ of $L_{i}$. Therefore, the time complexity of each iteration of the while loop is $O\left(n_{i} e_{i}\right)$. As a result, the time complexity of Algorithm 5 is $O\left(n_{i}^{2} e_{i}\right)$.

Next, we analyze the time complexity of Algorithm 4 using our heuristic algorithm. Given a loop nest $L$, in the worst case, this algorithm processes all loops of $L$. For each loop $L_{i}$ of $L$, Algorithm 4 calls Algorithm 3 to find the candidates of locked cache contents of $L_{i}$, and each call takes $\left.O\left(m_{i} n_{i} e_{i}+m_{i} n_{i}^{2} \log n_{i}\right)\right)$ time as discussed in Section 5 , where $m_{i}, n_{i}$, and $e_{i}$ are the number of memory blocks of $L_{i}$, the number of nodes, and the number of edges in the weighted DAG $G_{i}$ of $L_{i}$, respectively. In addition, for each cache set $y(y=0,1, \ldots, k-1)$, it calls Algorithm 5 to select a subset of the memory blocks in $C_{y}\left(L_{i}\right)$ and a set $M_{y}\left(L_{i}\right)$ of the previously selected memory blocks whose loading points are at the preheaders of the children of $L_{i}$ and lock the selected memory blocks at the preheader of $L_{i}$. Therefore, the time complexity of Algorithm 4 is $\left.\sum_{L_{i} \in L}\left(O\left(m_{i} n_{i} e_{i}+m_{i} n_{i}^{2} \log n_{i}\right)\right)+O\left(n_{i}^{2} e_{i}\right)\right)=O\left(d\left(m n e+m n^{2} \log n\right)\right)$, where $d, m$, $n$, and $e$ are the height of the loop nest tree of $L$, the number of memory blocks of $L$, the number of nodes, and the number of edges of the weighted graph of $L$, respectively. The height of the loop nest tree of $L$ is a very small number and can be considered as a constant. As a result, the time complexity of Algorithm 4 is $O\left(m n e+m n^{2} \log n\right)$. 


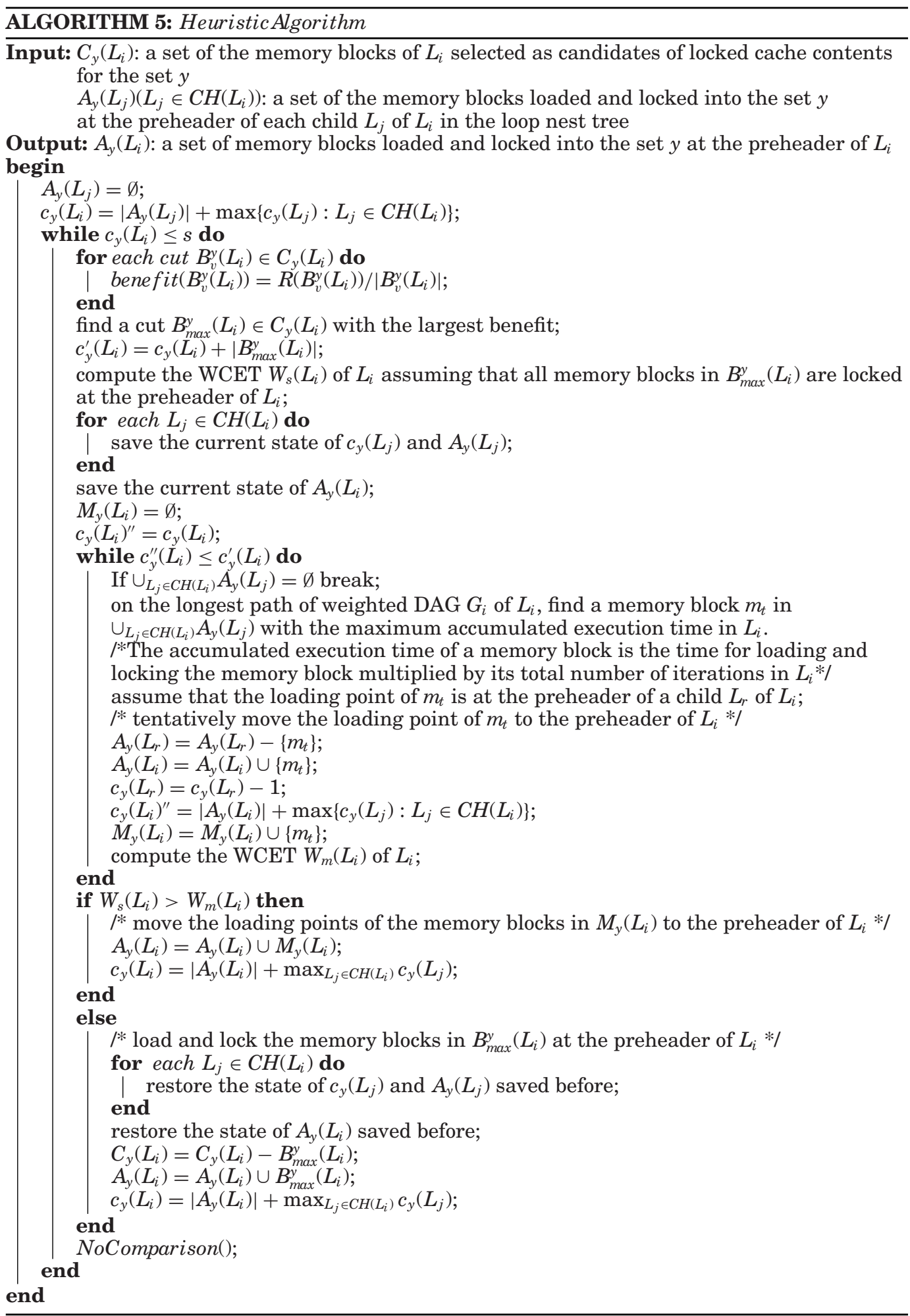




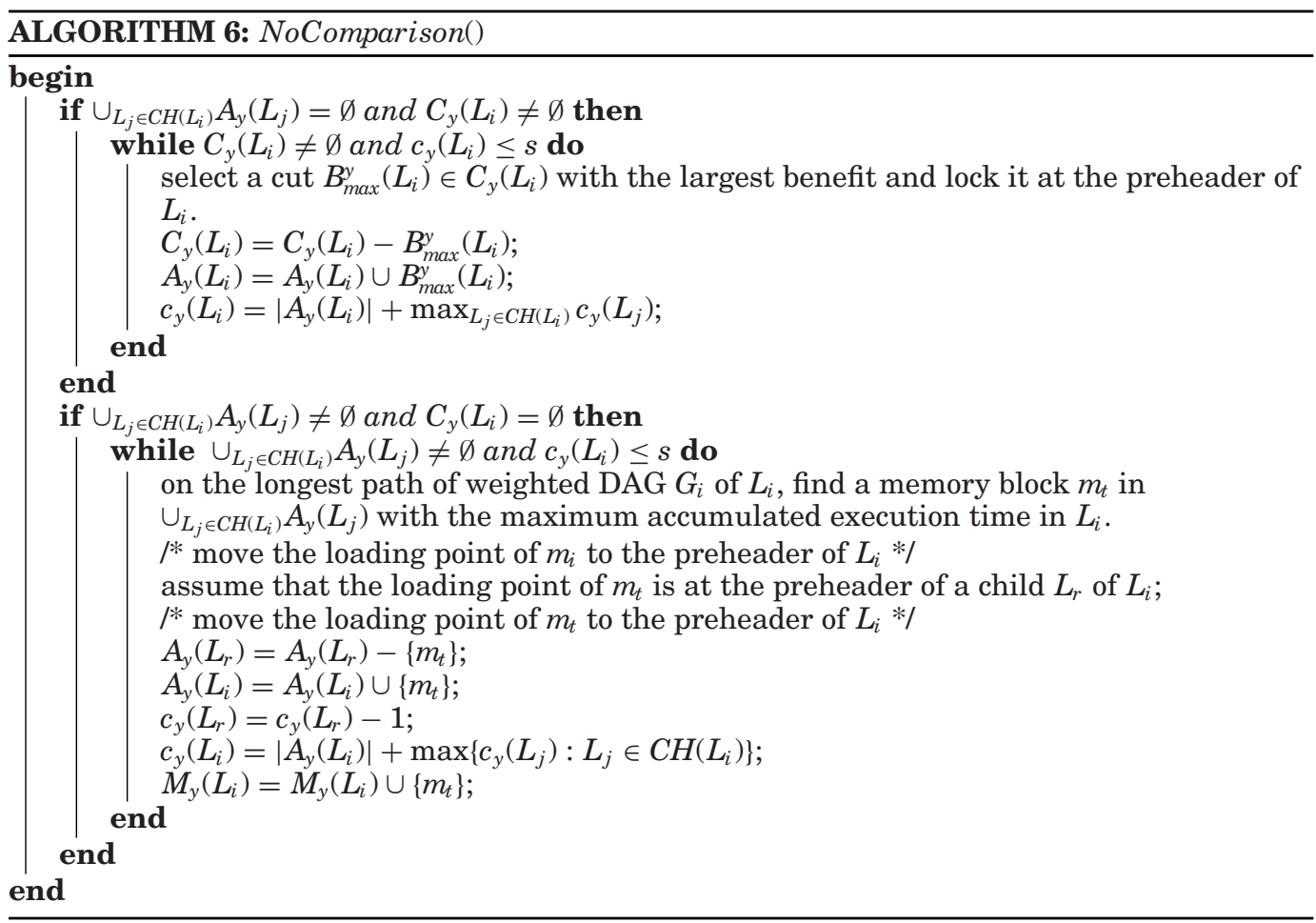

\subsection{ILP-Based Algorithm}

The objective of our ILP-based algorithm is the same as that of our heuristic algorithm. To minimize the WCET of $L_{i}$, we minimize the longest path length of the weighted graph $G_{i}$ of $L_{i}$.

For each memory block $m_{k}$ currently locked at the preheader of a child loop $L_{r}$ of the loop nest $L_{i}$ in the loop nest tree, we introduce a binary decision variable $X_{k}\left(L_{r}\right)$ to determine if $m_{k}$ is locked at the preheader of $L_{r}$ or the preheader of $L_{i}$ as follows:

$$
X_{k}\left(L_{r}\right)=\left\{\begin{array}{l}
1 \text { if } m_{k} \text { is locked at the preheader of } L_{i} \\
0 \text { if } m_{k} \text { is locked at the preheader of } L_{r}
\end{array} .\right.
$$

For each cut $B_{v}^{y}\left(L_{i}\right)$ of the loop $L_{i}$, we define a binary decision variable $Y_{v}^{y}\left(L_{i}\right)$ to determine whether the memory blocks in $B_{v}^{y}\left(L_{i}\right)$ are locked at the preheader of $L_{i}$ or not:

$$
Y_{v}^{y}\left(L_{i}\right)=\left\{\begin{array}{c}
1 \text { if all the memory blocks in } B_{v}^{y}\left(L_{i}\right) \text { are locked } \\
0 \quad \text { if no memory block in } B_{v}^{y}\left(L_{i}\right) \text { is locked }
\end{array} .\right.
$$

For each memory block $m_{t}$ of $L_{i}$, we introduce a binary variable to indicate if $m_{t}$ is locked at the preheader of $L_{i}$ as follows:

$$
Z\left(m_{t}\right)=\left\{\begin{array}{l}
1 \text { if } m_{t} \text { is locked at the preheader of } L_{i} \\
0 \quad \text { if } m_{i} \text { is not locked }
\end{array}\right.
$$

If $m_{t}$ is not in any cut of $C_{y}\left(L_{i}\right)$, we have the following constraint:

$$
Z\left(m_{t}\right)=0 .
$$


If $m_{t}$ is in a cut $B_{v}^{y}\left(L_{i}\right)$ of $C_{y}\left(L_{i}\right)$, we have the following constraint:

$$
Z\left(m_{t}\right)=Y_{v}^{y}\left(L_{i}\right)
$$

Next, we derive all other constraints.

6.3.1. Execution Time Constraints. For each node $v_{s}$ of $L_{i}, v_{s}$ is either a preheader node, a loop node, or a basic block node. Next, we derive the WCET of $v_{s}$, denoted by $w\left(v_{i}\right)$, for each of the three cases.

If $v_{s}$ is the preheader node of a child $L_{r}$ of $L_{i}$ in the loop nest tree, its weight $w\left(v_{s}\right)$ in $G_{i}$ is calculated as follows:

$$
w\left(v_{s}\right)=\sum_{m_{k} \in A_{y}\left(L_{r}\right)}\left(1-X_{k}\left(L_{r}\right)\right) * c * I_{i} .
$$

If $v_{s}$ is the preheader node of $L_{i}$, its weight in $G_{i}$ is formulated as follows:

$$
w\left(v_{s}\right)=\sum_{L_{r} \in C H\left(L_{i}\right)} \sum_{m_{k} \in A_{y}\left(L_{r}\right)} X_{k}\left(L_{r}\right) * c+\sum_{v=1}^{\lambda}\left(Y_{v}^{y}\left(L_{i}\right) *\left|B_{v}^{y}\left(L_{i}\right)\right|\right) * c,
$$

where $\left|B_{v}^{y}\left(L_{i}\right)\right|$ is the number of memory blocks in $B_{v}^{y}\left(L_{i}\right)$ and $\lambda$ is the number of minimum node cuts of the weighted graph $G_{i}$ of $L_{i}$ found by Algorithm 3 for the cache set $y$.

If $v_{s}$ is a loop node, its weight is a fixed value. If $v_{s}$ is a basic block node, its weight is computed as follows:

$$
w\left(v_{s}\right)=w_{s}-\sum_{m_{t} \in B\left(v_{s}\right)} Z\left(m_{t}\right) * \alpha\left(m_{t}\right),
$$

where $w_{s}$ is the execution time of the corresponding basic block of the node $v_{s}$ before locking the memory blocks of $v_{s}, B\left(v_{s}\right)$ is a set of the memory blocks the corresponding basic block of $v_{s}$ contains, and $\alpha\left(m_{t}\right)$ is the execution time reduction of the corresponding basic block of $v_{s}$ after locking $m_{t}$ into the I-cache.

6.3.2. Control Flow Constraints. The control flow constraints are constructed by using the weighted DAG $G_{i}$ of the loop $L_{i}$. We model the upper bound of the WCET of $L_{i}$ as follows:

(1) $G^{\prime}\left(L_{i}\right)=G\left(L_{i}\right)$.

(2) Add a dummy sink node $v_{e}$ and a directed edge from each of the previous sink nodes to the dummy sink node in $G^{\prime}\left(L_{i}\right)$.

(3) Set the weight of the dummy sink node $w\left(v_{e}\right)$ to 0.

(4) Let $t\left(v_{i}\right)$ be the length of the longest path from the node $v_{i}$ to the dummy sink node $v_{e}$ in $G^{\prime}\left(L_{i}\right)$.

(5) For the dummy sink node $v_{e}, t\left(v_{e}\right)=0$.

(6) For each node $v_{i} \neq v_{e}$, we have the following constraint:

$$
t\left(v_{i}\right) \geq \max \left\{t\left(v_{j}\right): v_{j} \text { is a child of } v_{i} \text { in the DAG }\right\}+w\left(v_{i}\right) .
$$

6.3.3. Set Capacity Constraints. Given a loop $L_{i}$, we build the set capacity constraints for each set $y$ as follows:

(1) For each child loop $L_{r}$ of $L_{i}$ in the loop nest tree, the number of memory blocks that are loaded and locked at the preheader of $L_{r}$ is formulated as follows:

$$
\left|A_{y}\left(L_{r}\right)\right|=\sum_{m_{k} \in A_{y}\left(L_{r}\right)}\left(1-X_{k}\left(L_{r}\right)\right)
$$


Table II. The Benchmarks

\begin{tabular}{|c|c|l|}
\hline Benchmark & Code Size (Bytes) & \multicolumn{1}{|c|}{ Description } \\
\hline cnt & 2,880 & Counting nonnegative numbers in the matrix \\
\hline crc & 5,168 & Cyclic redundancy check computation on 40 bytes of data \\
\hline edn & 10,563 & Finite impulse response filter calculations \\
\hline matmult & 3,737 & Matrix multiplication of two $20 \times 20$ matrices \\
\hline qurt & 4,898 & Root computation of quadratic equations \\
\hline adpcm & 26,852 & Adaptive pulse code modulation algorithm \\
\hline fir & 13,881 & Finite impulse response filter over a 700-item-long sample \\
\hline ud & 6,380 & Loop nesting of three levels \\
\hline select & 4,494 & Function to select the $n$-th largest number in a floating point array \\
\hline qsort-exam & 4,535 & Nonrecursive version of the quick sort algorithm \\
\hline nsichneu & 118,351 & Simulating an extended Petri Net \\
\hline statemate & 52,618 & Generated by the STAtechart Real-Time-Code generator STARC \\
\hline
\end{tabular}

(2) The total number of the cache lines in each set $y$ needed to store all selected memory blocks of loops rooted at $L_{r}$ is formulated as follows:

$$
c_{y}\left(L_{r}\right)=\left|A_{y}\left(L_{r}\right)\right|+\max _{L_{t} \in C H\left(L_{r}\right)} c_{y}\left(L_{t}\right),
$$

where $c_{y}\left(L_{t}\right)$ denotes the total number of the cache lines needed to store the selected memory blocks of the loop nest rooted at a child loop $L_{t}$ of $L_{r}$. Note that $c_{y}\left(L_{t}\right)$ is a fixed value.

(3) Similarly, we have the following constraints for the memory blocks that are loaded and locked at the preheader of $L_{i}$ :

$$
\begin{gathered}
\left|A_{y}\left(L_{i}\right)\right|=\sum_{L_{r} \in C H\left(L_{i}\right)} \sum_{m_{k} \in A_{y}\left(L_{r}\right)} X_{k}\left(L_{r}\right)+\sum_{v=1}^{\lambda}\left(Y_{v}^{y}\left(L_{i}\right) *\left|B_{v}^{y}\left(L_{i}\right)\right|\right), \\
c_{y}\left(L_{i}\right)=\left|A_{y}\left(L_{i}\right)\right|+\max _{L_{r} \in C H\left(L_{i}\right)} c_{y}\left(L_{r}\right) .
\end{gathered}
$$

(4) Last, we have the following set capacity constraint for the loop $L_{i}$ :

$$
c_{y}\left(L_{i}\right) \leq s
$$

6.3.4. Objective Function. Our ILP-based algorithm aims at minimizing the longest path length of $G_{i}$. Therefore, the objective function is formulated as follows:

$$
\min t\left(v_{r}\right)
$$

where $v_{r}$ is the preheader node of the loop $L_{i}$.

\section{EXPERIMENTAL RESULTS}

We have implemented our approach and compared it to the partial locking approach proposed by Ding et al. [2012] and the longest path-based dynamic cache locking approach proposed by Puaut [2006].

\subsection{Setup}

The benchmarks, as shown in Table II, are taken from the MRTC benchmark suite [Gustafsson et al. 2010]. The SimpleScalar PISA instruction set [Burger and Austin 1997] is used to compile benchmarks. We use Chronos [Li et al. 2007] to calculate the WCET of a task. Chronos implements the approach in Li et al. [2006] to estimate the WCET of a task. It models the WCET of a task using an ILP formulation. For instruction 
cache modeling, it employs a categorization-based technique proposed in Theiling et al. [2000] to classify instruction accesses. We switch off the branch prediction model of Chronos.

We use two sets of benchmarks. The first set contains 10 benchmarks with relatively smaller code sizes and loop nest sizes. For the benchmarks of the first set, we use two different associativities 2 and 4; two different cache line sizes 32B and 64B; and three different cache sizes $256 \mathrm{~B}, 512 \mathrm{~B}$, and $1 \mathrm{~KB}$. The miss latency is 30 cycles, and the hit latency is 1 cycle. Since the code sizes of the benchmarks of the first set are small, we choose small cache sizes. The second set contains three benchmarks with large code sizes and loop nest sizes. For the benchmarks of the second set, the cache structure used in our experiments is similar to that of the processor PowerPC 604e [Motorola 1996]. The cache size, the associativity, the cache line size, and the miss latency are $16 \mathrm{~KB}, 4$, $32 \mathrm{~B}$, and 38 cycles, respectively, as in PowerPC 604e. In addition, we use another two different cache sizes, $4 \mathrm{~KB}$ and $8 \mathrm{~KB}$, with the same cache associativity and cache line size. The loop nests of adpcm are much smaller than 16KB. Therefore, we artificially add an outer loop to the benchmark $a d p c m$, which contains the whole adpcm, and set the number of iterations of the outer loop to 10 .

We use MATLAB linprog as the ILP solver and run the programs for performing all experiments on an Intel i5-2430M CPU with $2.4 \mathrm{GHz}$ and $4 \mathrm{~GB}$ memory. The maximum running time of our approach is 46.6 seconds for the benchmark nsichneu, which has the largest code size. We also evaluate the impact of our approach on the code size. The largest percentage of the increased code size is $2.7 \%$ for all benchmarks. Notice that for a benchmark, the percentage of the increased code size is nearly linearly proportional to the ratio of the number of the selected memory blocks to the total number of the memory blocks of the benchmark. All experimental results are shown in Figures 10 through 17, where each horizontal axis denotes benchmarks and each vertical axis shows the improvements of our approach.

\subsection{Our Approach Versus Partial Locking}

For the first benchmark set, the experimental results show that our approach achieves the average improvements of $35 \%, 16 \%$, and $9 \%$ for the $256 \mathrm{~B}, 512 \mathrm{~B}$, and $1 \mathrm{~KB} 4$-way set-associative caches with a cache line size of $32 \mathrm{~B}$, respectively. For the $256 \mathrm{~B}, 512 \mathrm{~B}$, and $1 \mathrm{~KB} 2$-way set-associative caches with a cache line size of $32 \mathrm{~B}$, the average improvements are $31 \%, 12 \%$, and $8 \%$, respectively. For the $256 \mathrm{~B}, 512 \mathrm{~B}$, and $1 \mathrm{~KB} 2$-way set-associative caches with a cache line size of $64 \mathrm{~B}$, the average improvements are $32 \%$, $18 \%$, and $9 \%$, respectively.

For the second benchmark set, our approach achieves the average WCET improvements of $3 \%, 8 \%$, and $11 \%$ for the $4 \mathrm{~KB}, 8 \mathrm{~KB}$, and $16 \mathrm{~KB}$ caches, respectively.

Compared to the partial locking approach, our approach performs much better for the cache with a relatively small size when the code sizes of the benchmarks are small. The reason is that in our approach, many memory blocks have disjoint live ranges and can be mapped into the same cache lines, whereas in the partial locking approach, all memory blocks have overlapping live ranges and cannot share cache lines. However, if the cache size is much smaller than the code size of a benchmark, our approach makes much lower improvements. The reason is that much fewer memory blocks can be selected to be locked into the I-cache when the cache size is much smaller than the code size of a benchmark. Thus, the execution time reduction of a benchmark is very small by using both our approach and the partial locking approach.

Our approach shows dramatic improvements for the benchmarks that contain sequentially executed nonnested loops such as crc. Obviously, the live ranges of those loops do not overlap. Therefore, memory blocks of different loops can share the cache in our approach. For the benchmarks containing many deeply nested loops such as 


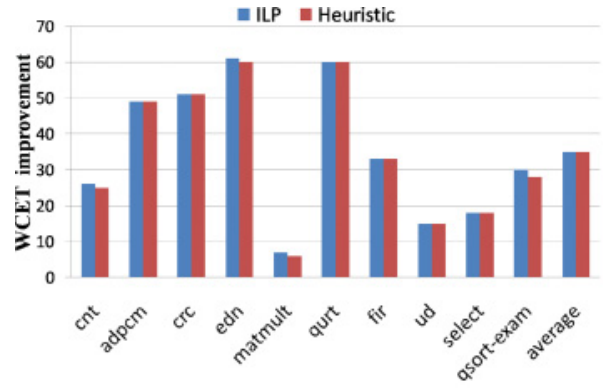

(a) cache size : $256 \mathrm{~B}$

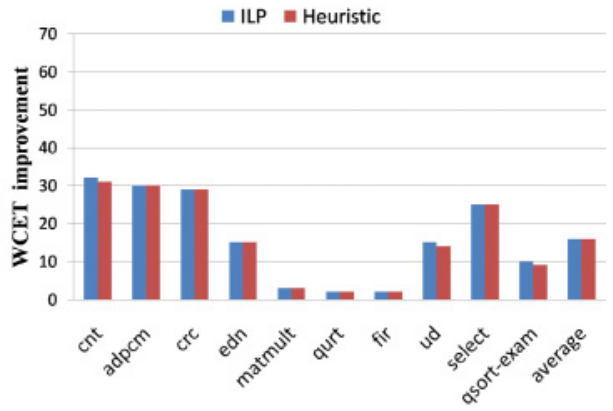

(b) cache size : $512 \mathrm{~B}$

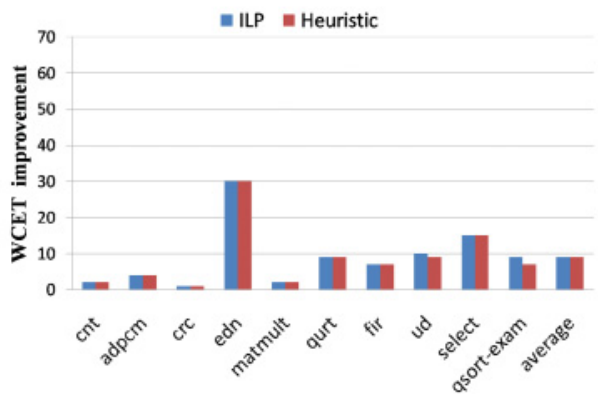

(c) cache size : $1 \mathrm{~KB}$

Fig. 10. WCET improvements of our approach over partial locking (4-way set associative caches with 32B cache lines).

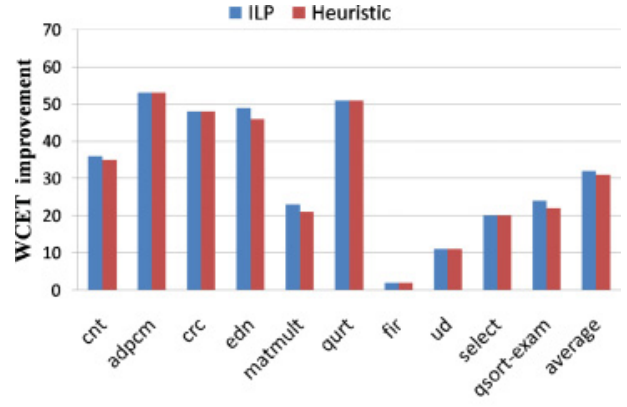

(a) cache size : 256B

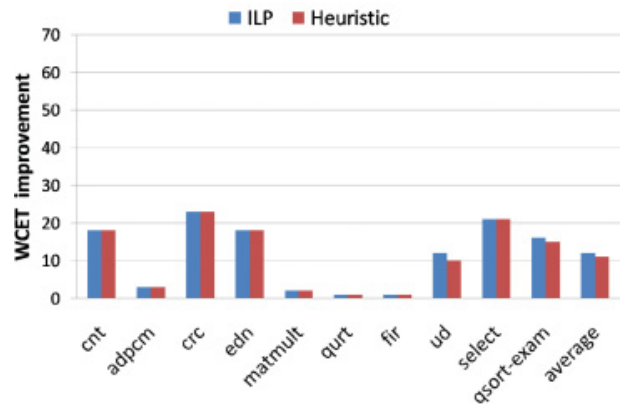

(b) cache size : 512B

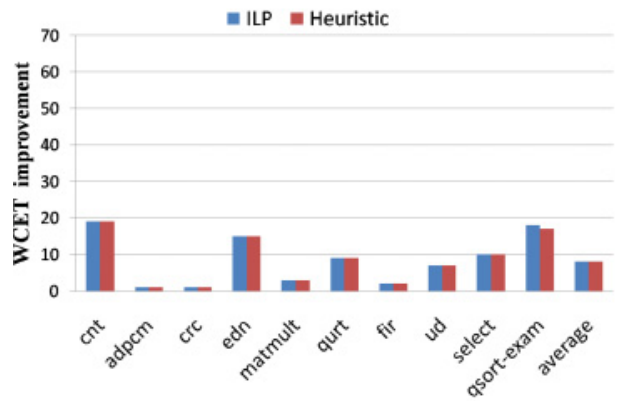

(c) cache size : $1 \mathrm{~KB}$

Fig. 11. WCET improvements of our approach over partial locking (2-way set-associative caches with 32B cache lines).

matmult, our approach shows lower improvements. The key reason is that in our approach, a lot of time is spent loading and locking the selected memory blocks.

The experimental results show that with a fixed cache line size, the improvement of our approach decreases as the associativity of the I-cache decreases. The key reason is that in our approach, fewer memory blocks can be loaded and locked into a set due to its decreased size. With a fixed associativity of the I-cache, the improvement of our approach increases as the cache line increases for the reason that our approach can lock more memory blocks into the cache due to the disjoint live ranges of locked memory blocks. 


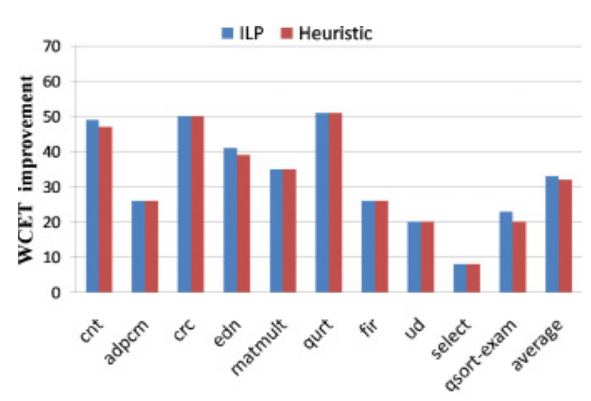

(a) cache size : $256 \mathrm{~B}$

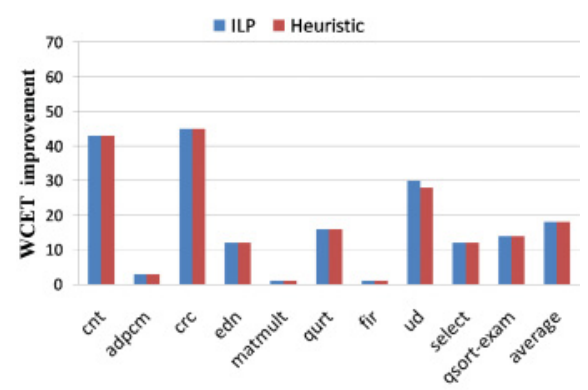

(b) cache size : $512 \mathrm{~B}$

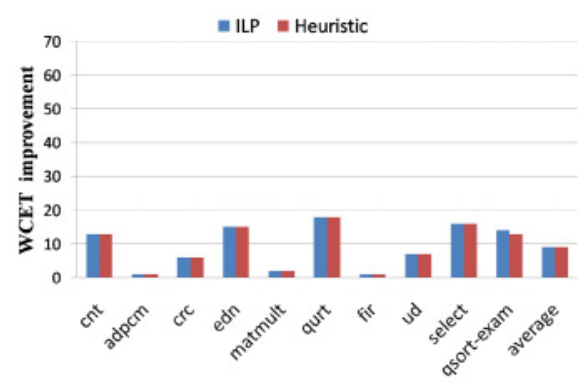

(c) cache size : $1 \mathrm{~KB}$

Fig. 12. WCET improvements of our approach over partial locking (2-way set-associative caches with 64B cache lines).

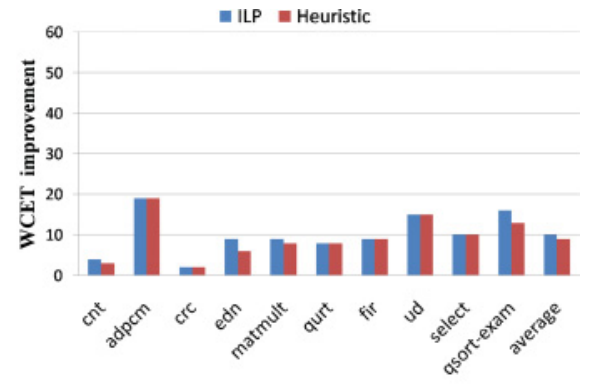

(a) cache size : $256 \mathrm{~B}$

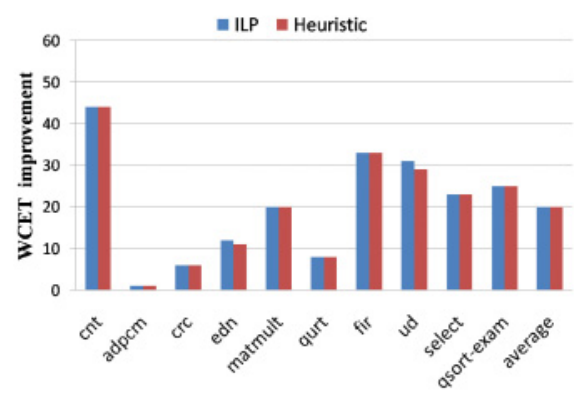

(b) cache size : $512 \mathrm{~B}$

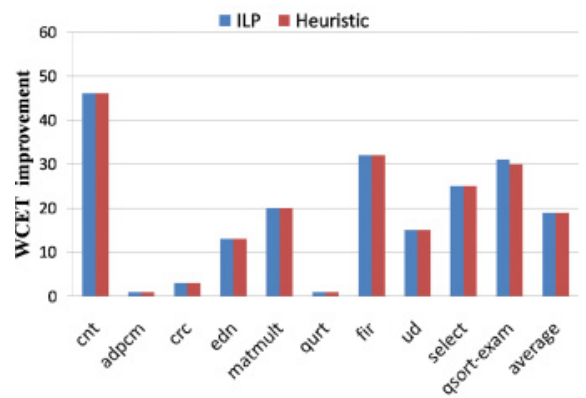

(c) cache size : $1 \mathrm{~KB}$

Fig. 13. WCET improvements of our approach over longest path-based dynamic locking (4-way setassociative caches with $32 \mathrm{~B}$ cache lines).

Overall, there are two major reasons that our approach performs significantly better than the partial locking approach. First, our approach uses dynamic cache locking so that memory blocks with different live ranges can be loaded and locked into the same set, resulting in more efficient utilization of the cache. Second, our approach selects a better set of memory blocks as locked cache contents by using the min-cut algorithm.

\subsection{Our Approach Versus Longest Path-Based Dynamic Locking}

Compared to the longest path-based dynamic locking approach, the experimental results of the first benchmark set show the average improvements of $9 \%, 20 \%$, and $19 \%$ for the $256 \mathrm{~B}, 512 \mathrm{~B}$, and $1 \mathrm{~KB} 4$-way caches with a cache line size of $32 \mathrm{~B}$, respectively. For the $256 \mathrm{~B}, 512 \mathrm{~B}$, and $1 \mathrm{~KB} 2$-way set-associative caches with a cache line size of $32 \mathrm{~B}$, 


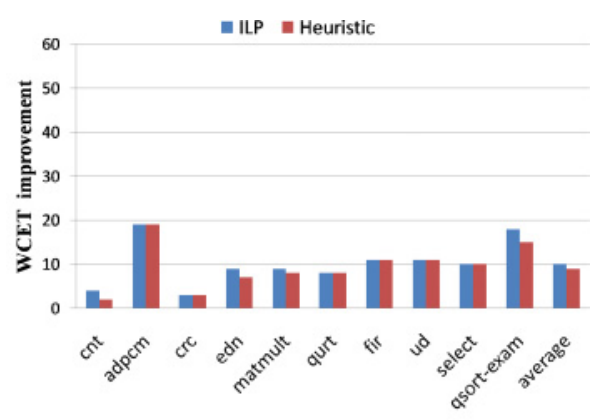

(a) cache size : $256 \mathrm{~B}$

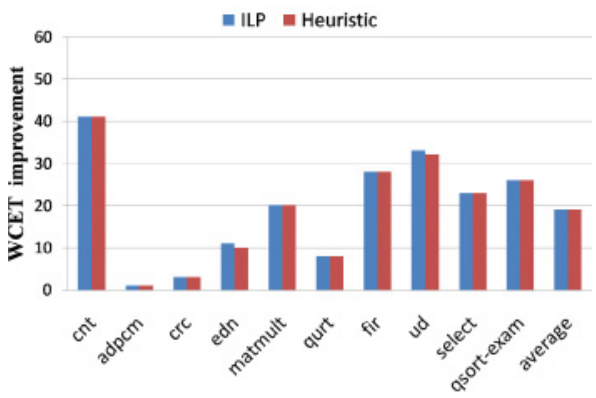

(b) cache size : $512 \mathrm{~B}$

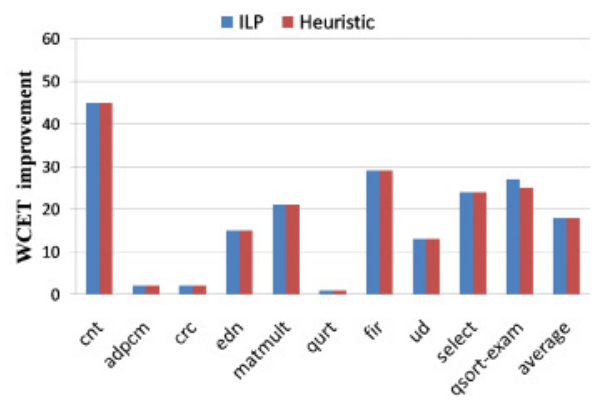

(c) cache size : $1 \mathrm{~KB}$

Fig. 14. WCET improvements of our approach over longest path-based dynamic locking (2-way setassociative caches with $32 \mathrm{~B}$ cache lines).

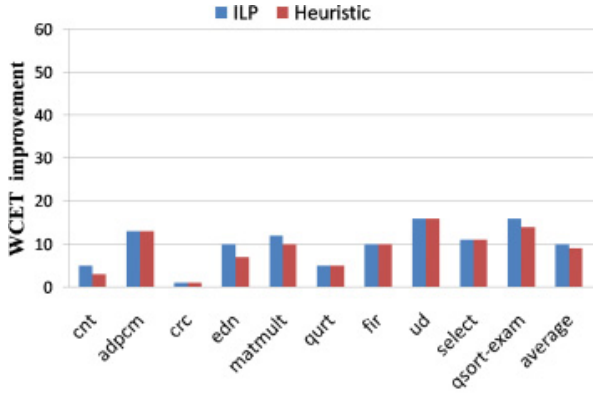

(a) cache size : $256 \mathrm{~B}$

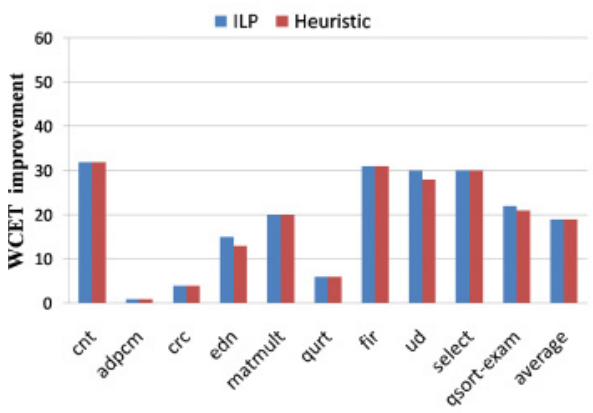

(b) cache size : $512 \mathrm{~B}$

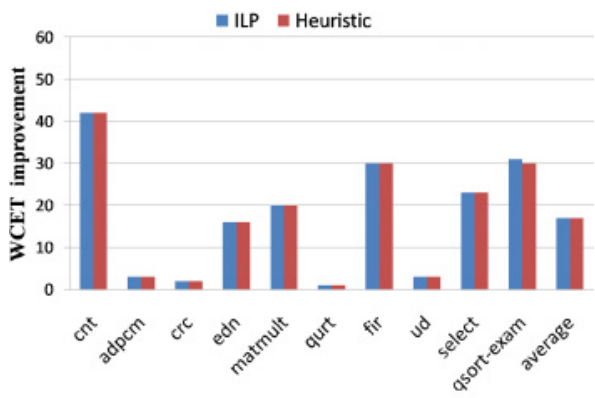

(c) cache size : $1 \mathrm{~KB}$

Fig. 15. WCET improvements of our approach over longest path-based dynamic locking (2-way setassociative caches with $64 \mathrm{~B}$ cache lines).

the improvements are $9 \%, 19 \%$, and $18 \%$, respectively. For the $256 \mathrm{~B}, 512 \mathrm{~B}$, and $1 \mathrm{~KB}$ 2 -way set-associative caches with a cache line size of $64 \mathrm{~B}$, the improvements are $9 \%$, $19 \%$, and $17 \%$, respectively.

For the second benchmark set, our approach achieves the average WCET improvements of $5 \%, 11 \%$, and $16 \%$ for the $4 \mathrm{~KB}, 8 \mathrm{~KB}$, and $16 \mathrm{~KB}$ caches, respectively.

Our approach preforms much better for the benchmarks that contain many deeply nested loops, such as matmult. The reason is that our approach moves the loading points of many memory blocks to the preheaders of outer loops to further reduce the execution frequencies of those lock instructions. For such benchmarks, the improvement increases as the cache size increases. The reason is that more selected memory 


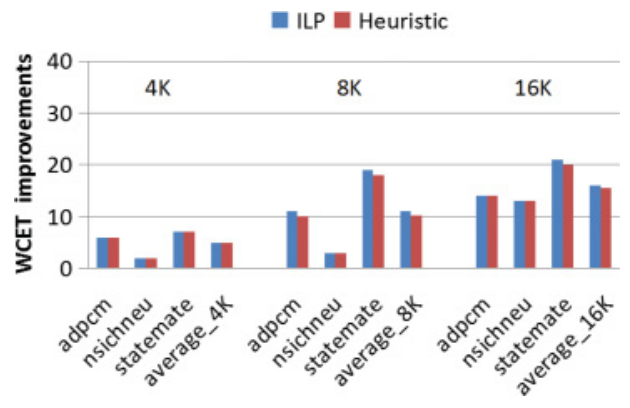

Fig. 16. WCET improvements of our approach over partial locking (4-way set-associative caches with sizes of $4 \mathrm{~KB}, 8 \mathrm{~KB}, 16 \mathrm{~KB}$, and $32 \mathrm{~B}$ cache lines).

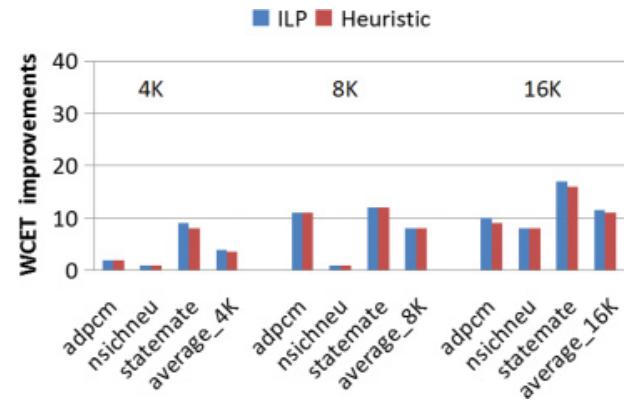

Fig. 17. WCET improvements of our approach over longest path-based dynamic locking (4-way setassociative caches with sizes of $4 \mathrm{~KB}, 8 \mathrm{~KB}, 16 \mathrm{~KB}$, and 32B cache lines).

blocks can be locked at the preheaders of outer loops. For the benchmarks that contain many sequentially executed nonnest loops such as crc, our approach shows lower improvements. The key reason is that each lock instruction is executed only once and the time on loading and locking instruction is nearly the same as the longest path-based locking approach.

When the cache size is much smaller than the code size of a benchmark, our approach makes lower improvements. The reason is that the WCET reduction is much smaller by using both our approach and the longest path-based approach when much fewer memory blocks can be locked into the cache.

On average, the results show similar improvement for different associativities and cache line sizes. With a fixed cache line size, the improvement of our approach slightly decreases as the associativity of the I-cache decreases for the reason that fewer memory blocks can be loaded and locked into a set in our approach. With a fixed associativity of the I-cache, the improvements remain similar.

Overall, our approach has two major advantages over the longest path-based dynamic locking approach. First, our approach selects a better set of memory blocks by using the min-cut algorithm. Second, our approach finds better loading points for the selected memory blocks.

\subsection{ILP-Based Algorithm Versus Heuristic Algorithm}

We have compared our heuristic algorithm and the ILP-based algorithm for finding a good loading point for each selected memory block. As the results show in Figures 10 through 15, our heuristic algorithm achieves nearly the same performance as the ILP-based algorithm. For the benchmarks that contain large nested loops such as qsort - exam, our ILP-based algorithm shows slightly better performance. For the benchmarks that only contain nonnested loops such as qurt, both algorithms show nearly the same performance.

\section{CONCLUSION}

In this article, we investigate the problem of selecting instructions of a task as locked cache contents to minimize the WCET of the task and propose a min-cut-based dynamic cache locking approach. Our approach considers the live ranges of memory blocks. Two memory blocks with disjoint live ranges can share the cache space, resulting in higher cache utilization. Our approach finds minimum cuts of the weighted DAG of each loop that contains not only the longest path but also the paths whose lengths are close to the longest path length. Therefore, our approach makes more efficient use of the I-cache 
than the previous approaches. Furthermore, we propose a heuristic algorithm and an ILP-based algorithm to find a good loading point for each selected memory block. The experimental results show that our approach achieves the average improvements of $33 \%, 15 \%, 9 \%, 3 \%, 8 \%$, and $11 \%$ over the partial locking approach proposed in Ding et al. [2012] for the $256 \mathrm{~B}, 512 \mathrm{~B}, 1 \mathrm{~KB}, 4 \mathrm{~KB}, 8 \mathrm{~KB}$, and $16 \mathrm{~KB}$ caches, respectively, and $9 \%, 19 \%, 18 \%, 5 \%, 11 \%$, and $16 \%$ over the dynamic locking approach proposed in Puaut [2006] for the $256 \mathrm{~B}, 512 \mathrm{~B}, 1 \mathrm{~KB}, 4 \mathrm{~KB}, 8 \mathrm{~KB}$, and $16 \mathrm{~KB}$ caches, respectively.

A typical embedded system consists of a set of tasks with various constraints, such as timing constraints. Constructing a feasible schedule satisfying all constraints is a primary goal at the design stage. Our future work is to integrate task scheduling and cache locking for multiprocessor-based embedded systems with two levels of caches. The major challenge is that the problem of selecting locked cache contents and the task scheduling problem are intertwined. On the one hand, we need to know the WCET of each task to construct a feasible schedule. On the other hand, we need to know how much cache space is allocated to each task to calculate its WCET. Given a schedule, for any two tasks, if their lifetimes overlap, they cannot share any space in both L1 caches and the L2 cache. Otherwise, they can share any space in both L1 caches and the L2 cache. The lifetime of a task is an interval where the start point is the start time of the task and the end point is the finish time of the task in the schedule. Given two tasks assigned to one processor, either the lifetime of one task contains the lifetime of the other task or the lifetimes of the two tasks are disjoint. This property leads to a polynomial-time optimal cache partitioning algorithm for all tasks sharing L1 caches. However, this property may not hold for two tasks that are assigned to two different processors. Since the L2 cache is shared by all tasks, the L2 cache partitioning problem becomes more complicated.

\section{ACKNOWLEDGMENTS}

We thank all reviewers for their insightful comments, which helped improve the quality of this article significantly.

\section{REFERENCES}

Kapil Anand and Rajeev Barua. 2009. Instruction cache locking inside a binary rewriter. In Proceedings of the Conference on Compilers, Architecture, and Synthesis for Embedded Systems. ACM, New York, NY, 185-194.

Kapil Anand and Rajeev Barua. 2015. Instruction-cache locking for improving embedded systems performance. ACM Transactions on Embedded Computing Systems 14, 3, Article No. 53.

Doug Burger and Todd M. Austin. 1997. The SimpleScalar tool set, version 2.0. ACM SIGARCH Computer Architecture News 25, 3, 13-25.

J. V. Busquets-Mataix, J. J. Serrano-Martin, R. Ors-Carot, P. Gil, and A. Wellings. 1996. Adding instruction cache effect to an exact schedulability analysis of preemptive real-time systems. In Proceedings of the 8th Euromicro Workshop on Real-Time Systems. IEEE, Los Alamitos, CA, 271-276.

A. M. Campoy, A. P. Ivars, and J. V. Busquets-Mataix. 2001. Using genetic algorithms in content selection for locking-caches. In Proceedings of the IASTED International Symposium on Applied Informatics. 271-276.

A. M. Campoy, A. P. Ivars, and J. V. Busquets-Mataix. 2002. Dynamic use of locking caches in multitask, preemptive real-time systems. IFAC Proceedings Volumes 35, 1, 411-416.

A. M. Campoy, A. Perles, F. Rodriguez, and J. V. Busquets-Mataix. 2003. Static use of locking caches vs. dynamic use of locking caches for real-time systems. In Proceedings of the Canadian Conference on Electrical and Computer Engineering (CCECE'03), Vol. 2. 1283-1286.

Huping Ding, Yun Liang, and Tulika Mitra. 2012. WCET-centric partial instruction cache locking. In Proceedings of the Design Automation Conference (DAC'12). IEEE, Los Alamitos, CA, 412-420.

Huping Ding, Yun Liang, and Tulika Mitra. 2014. WCET-centric dynamic instruction cache locking. In Proceedings of the 2014 Design, Automation, and Test in Europe Conference and Exhibition (DATE'14). $1-6$. 
Heiko Falk, Sascha Plazar, and Henrik Theiling. 2007. Compile-time decided instruction cache locking using worst-case execution paths. In Proceedings of the 5th IEEE/ACM International Conference on Hardware/Software Codesign and System Synthesis (CODES+ISSS'07). ACM, New York, NY, 143-148.

Jan Gustafsson, Adam Betts, Andreas Ermedahl, and Björn Lisper. 2010. The Mälardalen WCET benchmarks: Past, present and future. In Proceedings of the 10th International Workshop on Worst-Case Execution Time Analysis (WCET'10). 136-146.

Xianfeng Li, Yun Liang, Tulika Mitra, and Abhik Roychoudhury. 2007. Chronos: A timing analyzer for embedded software. Science of Computer Programming 69, 1-3, 56-67.

Xianfeng Li, Abhik Roychoudhury, and Tulika Mitra. 2006. Modeling out-of-order processors for WCET analysis. Real-Time Systems 34, 3, 195-227.

Yun Liang and Tulika Mitra. 2010. Instruction cache locking using temporal reuse profile. In Proceedings of the 47th Design Automation Conference (DAC'10). ACM, New York, NY, 344-349.

Tiantian Liu, Minming Li, and Chun Jason Xue. 2009. Minimizing WCET for real-time embedded systems via static instruction cache locking. In Proceedings of the 15th IEEE Real-Time and Embedded Technology and Applications Symposium (RTAS'09). IEEE, Los Alamitos, CA, 35-44.

Tiantian Liu, Minming Li, and Chun Jason Xue. 2012. Instruction cache locking for embedded systems using probability profile. Journal of Signal Processing Systems 69, 2, 173-188.

Tiantian Liu, Yingchao Zhao, Minming Li, and Chun Jason Xue. 2010. Task assignment with cache partitioning and locking for WCET minimization on MPSoC. In Proceedings of the 2010 39th International Conference on Parallel Processing (ICPP'10). IEEE, Los Alamitos, CA, 573-582.

Motorola. 1996. PowerPC 604e RISC Microprocessor Technical Summary. Retrieved February 10, 2017, from http://www.nxp.com/assets/documents/data/en/data-sheets/MPC604E.pdf.

Sascha Plazar, Jan C. Kleinsorge, Peter Marwedel, and Heiko Falk. 2012. WCET-aware static locking of instruction caches. In Proceedings of the Symposium on Code Generation and Optimization. 44-52.

Isabelle Puaut. 2006. WCET-centric software-controlled instruction caches for hard real-time systems. In Proceedings of the 2006 18th Euromicro Conference on Real-Time Systems. IEEE, Los Alamitos, CA, 217-226.

Isabelle Puaut and Alexis Arnaud. 2006. Dynamic instruction cache locking in hard real-time systems. In Proceedings of the 14th International Conference on Real-Time and Network Systems.

Isabelle Puaut and David Decotigny. 2002. Low-complexity algorithms for static cache locking in multitasking hard real-time systems. In Proceedings of the Real-Time Systems Symposium. IEEE, Los Alamitos, CA, 114-123.

Keni Qiu, Mengying Zhao, Chun Jason Xue, and Alex Orailoglu. 2014. Branch prediction-directed dynamic instruction cache locking for embedded systems. ACM Transactions on Embedded Computing Systems 13, 5s, Article No. 156.

Steven S. Skiena. 1998. The Algorithm Design Manual. Springer.

Mechthild Stoer and Frank Wagner. 1997. A simple min-cut algorithm. Journal of the ACM 44, 4, 585-591.

Henrik Theiling, Christian Ferdinand, and Reinhard Wilhelm. 2000. Fast and precise WCET prediction by separated cache and path analyses. Real-Time Systems 18, 2-3, 157-179.

Xavier Vera, Björn Lisper, and Jingling Xue. 2003. Data cache locking for higher program predictability. ACM SIGMETRICS Performance Evaluation Review 31, 1, 272-282.

Wenguang Zheng and Hui Wu. 2014. WCET-aware dynamic instruction cache locking. In Proceedings of the Conference on Languages, Compilers, and Tools for Embedded Systems. 53-62.

Wenguang Zheng and Hui Wu. 2015. WCET-aware dynamic D-cache locking for a single task. In Proceedings of the Conference on Languages, Compilersm and Tools for Embedded Systems. Article No. 8.

Wenguang Zheng and Hui Wu. 2017. Dynamic data-cache locking for minimizing the WCET of a single task. ACM Transactions on Embedded Computing Systems 16, 2, Article No. 31.

Received May 2016; revised November 2016; accepted December 2016 